\title{
Generalized Time- and Transfer-Constant Circuit Analysis
}

\author{
Ali Hajimiri \\ (Invited Paper)
}

\begin{abstract}
The generalized method of time and transfer constants is introduced. It can be used to determine the transfer function to the desired level of accuracy in terms of time and transfer constants of first-order systems using exclusively low frequency calculations. This method can be used to determine the poles and zeros of circuits with both inductors and capacitors. An inductive proof of this generalized method is given which subsumes special cases, such as methods of zero- and infinite-value time constants. Several important and useful corollaries of this method are discussed and several examples are analyzed.
\end{abstract}

Index Terms-Bandwidth enhancement techniques, circuit Analysis, Cochran-Grabel method, determination of poles and zeros, infinite-value time constants (IVT), method of time and trąnsfer constants (TTC), zero-value time constants (ZVT)

\section{INTRODUCTION}

A NALOG circuit design depends on analysis as a beacon to provide qualitative and quantitative input on how we can improve circuit performance by modifying its topology and/or parameters. A great deal of effort goes into improving the accuracy of device models and circuit simulators to predict the expected experimental outcome accurately on a computer before testing it in the lab. However, these absolutely necessary tools are not sufficient for analog circuit design, which by its nature is open-ended and divergent. This necessitates analytical techniques that can provide insight into how and where the circuit can be modified for design purposes.

The identification of the dominant source of a problem is at the core of design as it focuses creative energy on critical parts of the circuit and more importantly identify what kind of modifications will improve it. Generally, this is done by reducing the analysis into smaller more straightforward calculations that allow one to arrive at progressively more accurate approximations without performing the full analysis.

Although mesh and nodal analysis provide a systematic framework to apply Kirchhoff's current and voltage laws (KCL and KVL) to the circuit problem and convert them to a linear algebra problem that can be solved numerically using a computer(e.g., works of Bode [1] and Guillemin [2]), they are

Manuscript received February 17, 2009; revised May 20, 2009; accepted July 08, 2009. First published November 24, 2009; current version published June 09, 2010. This paper was recommended by Associate Editor W. A. Serdijn.

The author is with the Department of Electrical Engineering, California Institute of Technology, Pasadena, CA 91125 USA (e-mail: hajimiri@caltech.edu).

Color versions of one or more of the figures in this paper are available online at http://ieeexplore.ieee.org.

Digital Object Identifier 10.1109/TCSI.2009.2030092 not effective design tools. The analysis must be carried to the end before approximate results can be obtained and even then it is hard to obtain design insight from the resultant algebraic expressions particularly in terms of identifying the dominant sources of problem and topological solutions to them. This need was recognized by some of the early works in this area, e.g., [3] and [4].

An early instance of an approach suitable for design is the method of open-circuit time constants (OCT) developed by Thornton, Searle, et al. in early 1960s [5]. The OCT was developed for lumped electronic circuits with capacitors as their sole energy storing (reactive) elements to estimate their bandwidth limitation. It states that the coefficient of the term linear in complex frequency, $s$, in the denominator of the transfer function is exactly equal to the sum of time constants associated with each capacitor alone when all other capacitors are open circuited and sources are nulled. The original derivation of the OCT [5] and its subsequent generalizations to both capacitors and inductors, namely the method of zero value time-constants (ZVT), was based on evaluation of the determinant of the $Y$ matrix in the nodal equations and how its co-factors change due to the capacitors [5]. The ZVT method is powerful since it provides a clear indication of the dominant source of bandwidth limitation and guidance into potential solutions. In Section IV, we present an alternative inductive derivation of ZVT's and will generalize the method by using transfer constants to account for the effect of zeros on the bandwidth estimate.

The approach used in [5] was generalized in early 1970s by Cochran and Grabel [6] to determine as many of the denominator coefficients as needed by calculating the time-constants associated with each reactive element under different combinations of shorting and opening of other reactive elements in the circuit. Unlike nodal analysis, this process can be stopped at any point when the desired level of accuracy for the denominator has been obtained. The notation was cleaned further in the 1980s by Rosenstark to express denominator coefficient only in terms of time constants in a systematic way [10]. In the 1990s, the method was generalized to include the effect of transcapacitors by Fox et al. [12] and mutual inductors by Andreani et al. [14].

In late 1970s, Davis developed a method for determination of the numerator (and thus the zeros) of the transfer functions of lumped RC circuits using a combination of the time-constants and the low frequency transfer functions under different combinations of shorting and opening of the capacitors [7]-[9]. We will discuss a generalization of this method with a more intuitive notation in Section V. 
The transfer function of a first-order system can also be determined using the extra element theorem presented in late 1980s by Middlebrook [11]. In this case, two of the three low frequency calculations are identical to Davis's approach [7]. The third calculation used to determine the numerator of the transfer function involves a null-double injection, which involves simultaneous usage of two sources to null the output signal. The approach was generalized to $N$ extra elements in late 1990s in [13] and [15], where the denominator of the transfer function is calculated the same way as the Cochron-Grabel method [6] and its numerator using multiple null double injections. The approach presented in this paper does not use the null double injection and provides a more intuitive link between the zeros and the time and transfer constants.

In Section II, we discuss some of the general properties of a transfer function. We find the general transfer function of a firstorder system in Section III, where the concept of transfer constant is defined. Next, we investigate an $N$ th-order system and derive its first-order numerator and denominator coefficients. We provide an inductive intuitive proof and a generalization of the method of zero-value time constants and its generalization for the numerator using the concept of transfer constant in Section IV. In Section V, we discuss how all the coefficients of the numerator and denominator can be calculated using the method of time and transfer constants (TTC) and thus provide a complete method to determine the transfer function to the desired level of accuracy. Some of the important corollaries to circuit design will be discussed in VI. Finally, Section VII provides several examples of the method, which will be referred to through the text.

\section{General Properties of Transfer FunCtions}

For a single-input single-output linear time-invariant (LTI) network, the transfer function can be defined as the ratio of the voltages and/or currents of any two arbitrary ports of the circuit, including the ratio of the voltage and current of the same port. We designate the input and output variables as $x$ and $y$. For example, when $x$ is an input voltage due to a voltage source and $y$ is the voltage of another node in the circuit, the transfer function, $H(s) \equiv v_{o}(s) / v_{i}(s)$, would correspond to a voltage gain. On the other hand, if the input, $x$, is the current of a current source driving a given port of the circuit, while the output, $y$, is the voltage across the same port, the transfer function, $Z(s) \equiv v_{1}(s) / i_{1}(s)$ would correspond to the impedance looking into that port. ${ }^{1}$

The transfer function of a linear system with lumped elements can be written as ${ }^{2}$

$$
H(s)=\frac{a_{0}+a_{1} s+a_{2} s^{2}+\ldots+a_{m} s^{m}}{1+b_{1} s+b_{2} s^{2}+\ldots+b_{n} s^{n}}
$$

\footnotetext{
${ }^{1}$ One has to be careful about the choice of stimulus and output. If a node is excited with a current source and the voltage across that node is measured, then the quantity measured is the impedance, $Z(s)$. On the other hand, if the same port is excited by a voltage source and its current is the output variable, the calculated transfer function is the admittance $Y(s)$. Although in the end, we must have $Z(s)=1 / Y(s)$, one should keep things consistent, as the poles of $Z(s)$ are the zeros of $Y(s)$ and vice versa. This is important in nulling the independent source, which means a short-circuit for a voltage source and an open-circuit for the current source.

${ }^{2}$ The leading one in the denominator is absent for transfer functions that go to infinity at dc (e.g., the input impedance of a capacitor to ground). In such cases, it is more straightforward to evaluate the inverse transfer function (e.g., admittance in the case of the capacitor).
}

where all $a_{i}$ and $b_{j}$ coefficients are real and $s$ represents the complex frequency. Coefficient $a_{0}$ is the low frequency (dc) transfer function. This equation can be factored as 3

$$
H(s)=a_{0} \cdot \frac{\left(1-\frac{s}{z_{1}}\right)\left(1-\frac{s}{z_{2}}\right) \ldots\left(1-\frac{s}{z_{m}}\right)}{\left(1-\frac{s}{p_{1}}\right)\left(1-\frac{s}{p_{2}}\right) \ldots\left(1-\frac{s}{p_{n}}\right)}
$$

where based on the fundamental theorem of algebra, the pole and zero frequencies $\left(p_{i}\right.$ and $\left.z_{i}\right)$ are either real or appear as complex conjugate pairs.

The order of the denominator, $n$, determines the number of natural frequencies of the system and is equal to the number of independent energy storage elements in the circuits. This is equal to the maximum number of independent initial conditions (capacitor voltages and inductor currents) that can be set, as we will see later in Section V. Natural frequencies of the circuit are independent of the choice of the input and output variables and are intrinsic characteristics of the circuit. ${ }^{4}$

On the contrary, the zeros of the transfer function, i.e., the roots of the numerator of (1), do depend on the choice of the input and output. While it is possible to answer what the poles of a circuit are without knowing what the input and output variables are, it is meaningless to ask the same question about the zeros, as they assume different values for different choices of the input and/or the output.

Knowing the coefficients of the transfer function of an LTI system (or equivalently its poles and zeros), we can predict its dynamics. In the following sections we see how we can determine the transfer function of an $N$ th-order system to the desired level of accuracy using low frequency calculations of port resistances and low-frequency values of the transfer functions (transfer constants) for different combinations of shorting and opening of other elements. We will start with first-order system similar to that in [7] with a modified, more generalizable notation.

\section{FIRST-ORDER SYSTEM}

Let us consider an LTI circuit with a single energy-storing element, an input $x$, and an output $y$, as shown in Fig. 1(a) and (b) for a system with a capacitor or an inductor, respectively. Although these circuits include only one reactive element, $C_{1}$ or $L_{1}$, the network in the box can be quite complex with any number of frequency-independent elements, such as resistors and dependent sources.

${ }^{3}$ The factorization of (1) is most suitable to describe a low-pass system. In the case of a band-pass amplifier with a well defined mid-band gain, $a_{\text {mid }}$, (where $a_{0}$ can be very small or even zero) the poles and the zeros can be divided into two groups: those occurring below mid-band and those that fall above it. To have a flat pass-in this case, the number of poles and zeros below mid-band must be equal. Thus, (2) can be reordered as (due to Middlebrook)

$$
H(s)=\frac{\left(1-\frac{z_{1}}{s}\right) \ldots\left(1-\frac{z_{k}}{s}\right)}{\left(1-\frac{p_{1}}{s}\right) \ldots\left(1-\frac{p_{k}}{s}\right)} \cdot a_{m i d} \cdot \frac{\left(1-\frac{s}{z_{k+1}}\right) \ldots\left(1-\frac{s}{z_{m}}\right)}{\left(1-\frac{s}{p_{k+1}}\right) \ldots\left(1-\frac{s}{p_{n}}\right) .}
$$

This representation is helpful when we try to separate the effect of the poles and zeros affecting the low cut-off frequency, $\omega_{l}$, from those controlling the high cut-off frequency, $\omega_{h}$.

${ }^{4}$ The implicit assumption here is that these modes are observable in the control theoretical sense. 

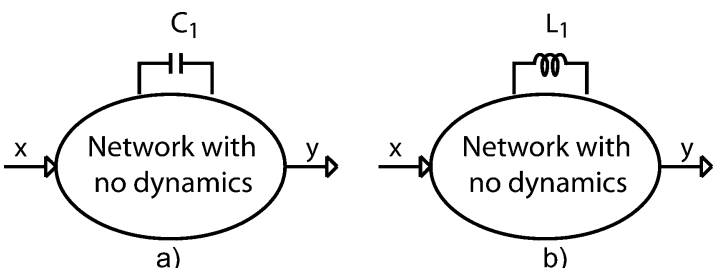

Fig. 1. First-order system with a) a capacitor as the energy storing element; b) an inductor.

A circuit with one reactive element has one pole and one zero. ${ }^{5}$ For a first-order system, (1) reduces to

$$
H(s)=\frac{a_{0}+a_{1} s}{1+b_{1} s}
$$

where $a_{0}$ is the low-frequency transfer function. The pole will be at $p=-1 / b_{1}$ corresponding to a pole time constant of $\tau \equiv b_{1}$. The zero occurs at $z=-a_{0} / a_{1}$.

Now we use transfer constants defined as low frequency transfer functions from the input to the output under different combinations of shorted and opened reactive elements (shown with capital $H$ with different superscripts) to determine of the transfer function. Our first transfer constant is the value of the transfer function when the reactive element (or in general all reactive elements) is (are) zero valued $(C=0$, i.e., open circuited capacitor and $L=0$, i.e., short circuited inductor). This transfer constant is designated as $H^{0}$. This is the same as the low frequency transfer function since setting every reactive element to zero removes any frequency dependence from the circuit, i.e.,

$$
a_{0}=H^{0} .
$$

For a first-order system with a capacitor, $C_{1}$, the only time constant, $\tau_{1}$, is simply $R_{1}^{0} C_{1}$, where $R_{1}^{0}$ is the resistance seen across the capacitor with all the independent sources (including the input) nulled. (Nulling a source means replacing an independent voltage source with a short circuit and an independent current source with an open-circuit.) Here, the circuit of Fig. 1(a) simply reduces to the parallel combination of capacitor, $C_{1}$, and the low frequency resistance it sees, $R_{1}^{0}$. Therefore, we have a pole time constant of

$$
\tau_{1} \equiv R_{1}^{0} C_{1}=b_{1}
$$

where the superscript zero in $R_{1}^{0}$ indicates that the independent sources and the energy-storing element are at their zero values and the subscript is the index of the energy storing element. Equivalently, if the reactive element is an inductor, $L_{1}$, the timeconstant is

$$
\tau_{1} \equiv \frac{L_{1}}{R_{1}^{0}}
$$

Let us continue with the case of capacitor for the time being. The impedance of the capacitor $C_{1}$ is simply $1 / C_{1} s$. We notice that the capacitance, $C_{1}$, and the complex frequency, $s$, always appear together as a product, so the transfer function of (3) can be unambiguously written as

$$
H(s)=\frac{a_{0}+\alpha_{1} C_{1} s}{1+\beta_{1} C_{1} s}
$$

${ }^{5}$ Sometimes, we say there is "no zero" when it is at infinity. where $\alpha_{1}$ and $\beta_{1}$ have the appropriate units. Combining (5) and (7), we obtain

$$
\beta_{1}=R_{1}^{0} \text {. }
$$

We can use another transfer constant to determine the numerator of (7). This time assume that the value of $C_{1}$ goes to infinity. For a capacitor this is equivalent to having it replaced with a short circuit. For $C_{1} \rightarrow \infty$, the second terms in the numerator and the denominator of the transfer function of (7) dominate, and, hence, it reduces to

$$
\left.H^{1} \equiv H\right|_{C_{1} \rightarrow \infty}=\frac{\alpha_{1}}{\beta_{1}}
$$

where $H^{1}$ is another transfer constant evaluated from the input $x$ to the output $y$ with the reactive element at its infinite value, e.g., capacitor $C_{1}$ short circuited. This is simply another frequency-independent gain calculation. Note that in general this transfer constant, $H^{1}$, is different from the first transfer constant, $H^{0}$, which is the low frequency transfer function with the energy storing element being zero valued (capacitor open circuited or inductor shorted).

Considering (8), (9), and comparing (3) with (7), we easily determine $a_{1}$ to be

$$
a_{1}=\alpha_{1} C_{1}=R_{1}^{0} C_{1} H^{1}=\tau_{1} H^{1}
$$

where $\tau_{1}$ is simply the pole time constant defined by (5) or (6).

The result of this derivation is that the transfer function of a system with one energy-storing element can be expressed as

$$
H(s)=\frac{H^{0}+\tau_{1} H^{1} s}{1+\tau_{1} s}
$$

where $H^{0}$ is the zero-valued transfer constant from the input, $x$, to the output $y$ when the reactive element is zero-valued $\left(C_{1}\right.$ opened or $L_{1}$ shorted), $H^{1}$ is the infinite-value transfer constant ( $C_{1}$ shorted or $L_{1}$ opened), and $\tau_{1}$ is the time constant associated with the reactive element and resistance it sees with the independent sources nulled, $R_{1}^{0}$. As can be seen for a single energy-storing element, (11) provides the exact transfer function of the system, in terms of three low-frequency calculations.

\section{Zero-VAlue Time AND TRANSFER CONSTANTS}

Having considered a system with one energy storage element, in this section we take the first step toward a complete generalization of the approach to the case with $N$ energy storing elements, which will be presented in Section V. We will start by determining the first term in the denominator and numerator of (1), namely, $b_{1}$ and $a_{1}$ in a system with $N$ reactive (energy-storing) elements. First, using an alternative inductive, more intuitive (as opposed to deductive), derivation than that of [5], we determine $b_{1}$ as the sum of the so-called zero-value time constants (ZVT) of the network. ${ }^{6} \mathrm{Next}$, we also derive a general expression for $a_{1}$ in terms of these ZVT's and some low frequency transfer functions.

\footnotetext{
${ }^{6}$ In [5], the first denominator term, $b_{1}$, for capacitors only is derived using an $n$-port nodal analysis of the above system and calculations of the co-factors of the circuit determinant. It is generalized in [6] using a similar matrix-based approach.
} 


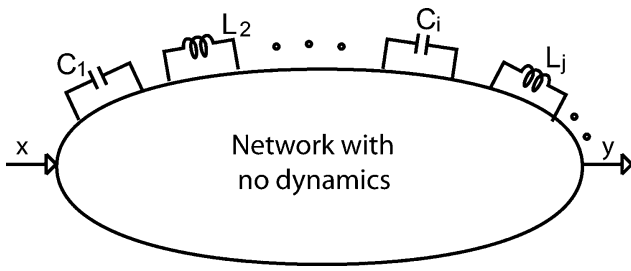

Fig. 2. Network with $N$ ports in addition to the input and output with all the inductors and capacitors presented at the additional ports and no energy storing element inside.

Any network with $N$ energy-storing (reactive) elements can be represented as a system with $N$ external ports (in addition to the input and output) with no frequency-dependent elements inside (e.g., containing only resistors and dependent voltage and current sources) and each reactive element (namely inductors and capacitors) attached to one of the ports, as shown in Fig. 2. (If more than one reactive element is connected to the same pair of terminals, each one of them is assumed to have a port of its own with a separate index.)

The only way for a coefficient $s$ to occur in a transfer function of a lumped circuit is as a multiplicative factor to a capacitor or an inductor, as in $C_{i} s$ or $L_{j} s$. Let us initially limit our discussion to just capacitors and then generalize to include the inductors. In that case, the $b_{1}$ coefficient in (1) must be a linear combination of all the capacitors in the circuit. Note that the $b_{1}$ term cannot contain a term $C_{i} C_{j}$ because such a term must have an $s^{2}$ multiplier. Applying the same line of argument, the $b_{2}$ coefficient must consist of a linear combination of two-way products of different capacitors $\left(C_{i} C_{j}\right)$, as they are the only ones that can generate an $s^{2}$ term. ${ }^{7}$ In general, the coefficient of the $s^{k}$ term must be a linear combination of nonrepetitive $k$-way products of different capacitors. The same argument can be applied to $a_{k}$ coefficients in the numerator and, hence, we can write the transfer function as

$H(s)=\frac{a_{0}+\left(\sum_{i=1}^{N} \alpha_{1}^{i} C_{i}\right) s+\left(\sum_{i}^{1 \leqslant i<j \leqslant N} \sum_{j}^{i j} \alpha_{2}^{i j} C_{i} C_{j}\right) s^{2}+\ldots}{1+\left(\sum_{i=1}^{N} \beta_{1}^{i} C_{i}\right) s+\left(\sum_{i}^{1 \leqslant i<j \leqslant N} \sum_{j}^{i j} \beta_{2}^{i j} C_{i}\right) s^{2}+\ldots}$

where coefficients $\alpha$ and $\beta$ have the appropriate units. Note that the double (and higher order) sums are defined in such a way to avoid redundancy due to the repetition of terms such as, $C_{i} C_{j}$ and $C_{j} C_{i}$. Also note that the superscripts " $i$ " and " $j$ " is used as an index and not an exponent.

The idea behind the derivation of $a_{i}$ and $b_{i}$ coefficients in general is to choose a set of extreme values (zero and infinity or equivalently open and short) for energy storing elements in such a way that we can isolate and express one of the $\alpha$ or $\beta$ parameters at a time in terms of other parameters we already know and low-frequency calculations involving no reactive elements at all.

\footnotetext{
${ }^{7}$ We will see in footnote 8 why they cannot be the same capacitor, i.e., $\left(C_{i}\right)^{2}$.
}

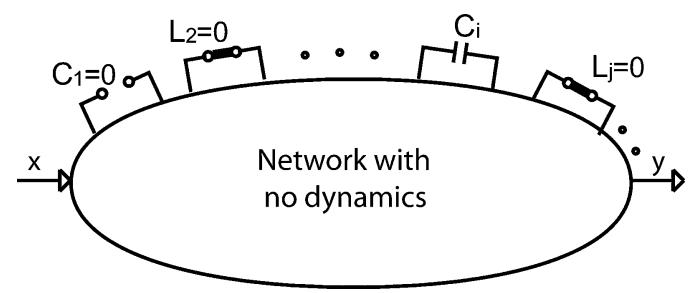

Fig. 3. Network with $N$ ports in addition to the input and output with with all the inductors and capacitors zero valued except $C_{i}$.

\section{A. Determination of $b_{1}$}

In this subsection, we show that $b_{1}$ is exactly equal to the sum of the zero-value time constants (ZVT) and thereby provide an alternative derivation of the ZVT method. The zero-value time constant for each reactive element is essentially the timeconstants of the first-order systems formed by forcing all other reactive elements to be at their zero values, i.e., open-circuited capacitors and shorted-circuited inductors.

The transfer function of (12) is determined independently of the specific value of the capacitor and must, therefore, be valid for all capacitor values including zero and infinity. To determine $b_{1}$, let us look at a reduced case when all capacitors, except $C_{i}$, have a value of zero, as depicted in Fig. 3. The transfer function of (12) with a single $C_{i}$ reduces to the following first-order one ${ }^{8}$

$$
H_{i}(s)=\frac{a_{0}+\alpha_{1}^{i} C_{i} s}{1+\beta_{1}^{i} C_{i} s} .
$$

We have already determined the transfer function of a general first-order system in (11). The reduced system of Fig. 3 is one such first-order system with a time constant of

$$
\tau_{i}^{0}=R_{i}^{0} C_{i}
$$

where $R_{i}^{0}$ is the resistance seen by the capacitor $C_{i}$ looking into port $i$ with all other reactive elements their zero value (hence, the superscript zero), namely open-circuited capacitors (and shortcircuited inductors), and the independent sources nulled. Equations (11), (13), and (14) clearly indicate that

$$
\beta_{1}^{i}=R_{i}^{0} .
$$

This argument is applicable to any capacitor in the system. Hence, the first denominator coefficient in (1), $b_{1}$, is simply given by the sum of these zero-value time constants $(Z V T)^{9}$

$$
b_{1}=\sum_{i=1}^{N} \tau_{i}^{0}
$$

${ }^{8}$ Here, we can see why the higher order terms in (12) cannot contain any self-product terms (e.g., $C_{i} \cdot C_{i}$ ) from Fig. 3. A $\left(C_{i}\right)^{2}$ term in the sums defining $a_{2}$ or $b_{2}$ in (12) would result in a second-order transfer function in (13) which contradicts the fact that the reduced system of Fig. 3 has only one energy storing element. By the same token, terms such as $\left(C_{i}\right)^{2} C_{j}$ cannot appear in higher order terms, such as $b_{3}$.

${ }^{9}$ This method is sometimes referred to as the method of open-circuit time (OCT) constants. This terminology only makes sense when applied to capacitors because a zero-valued capacitor corresponds to an open circuit. Unfortunately, an inductor at its zero value corresponds to a short circuit, and, thus, the name becomes misleading. 
where $\tau_{i}^{0}$ coefficients are the ZVT's given by (14) for capacitors. With both inductors and capacitors present, the summation terms in (12) will be linear combination of inductors and capacitors and sums of their products for higher order terms. With an inductor $L_{j}$ at port $j$, setting all the other elements to their zero value and nulled independent sources, the system reduces to yet another first-order system with a time constant similar to (6), i.e.,

$$
\tau_{j}^{0}=\frac{L_{j}}{R_{j}^{0}}
$$

Hence, in general, the $\tau_{i}^{0}$ terms are zero-value time constants associated with the capacitor or the inductor given by (14) or (17).

Note that the sum of zero-value time constants in (16) is exactly equal to the sum of pole characteristic times $\left(-1 / p_{i}\right)$ which is also equal to $b_{1}$, as can be easily seen by comparing (1) and (2). However, it is very important to note that in general ${ }^{10}$ there is no one-to-one correspondence between the individual zero-value time constant, $\tau_{i}$, and pole frequency, $p_{i}$. (For one thing, the individual poles can be complex while the time constants are always real. Also, as we will see in Section VI-A, the number of the poles and the number of time constants are not necessarily the same.)

\section{B. Determination of $a_{1}$}

Next, we determine the numerator coefficient $a_{1}$, which can be used to approximate the effect of the zeros. We will see that $a_{1}$ can be written in terms of the zero-value time constants already determined in calculation of $b_{1}$ and low-frequency transfer constants evaluated with one reactive element infinite-valued at a time. We rely on the first-order system result of Section III to determine the $\alpha_{1}^{i}$ coefficients in (12).

When $C_{i} \rightarrow \infty$ in (13) while the other elements are still at zero value, (Fig. 4) the transfer function from the input to the output reduces to a constant, i.e.,

$$
\left.H^{i} \equiv H\right|_{\substack{C_{i} \rightarrow \infty \\ c_{j=0}=0 \\ i \neq j}}=\frac{\alpha_{1}^{i}}{\beta_{1}^{i}}
$$

where $H^{i}$ is a first-order transfer constant between the input and the output with the single reactive element $i$ at its infinite value (i.e., short circuited capacitor or open circuited inductor) and all others zero-valued.11 We have already determined $\beta_{1}^{i}$ to be $R_{i}^{0}$ in (15), which leads to $\alpha_{1}^{i}=R_{i}^{0} H^{i}$ from (18). Therefore, $\alpha_{1}^{i} C_{i}=R_{i}^{0} C_{i} H^{i}=\tau_{i}^{0}$. Thus, we can write

$$
a_{1}=\sum_{i=1}^{N} \tau_{i}^{0} H^{i}
$$

which is the sum of the products of zero-value time constants given by (14) or (17) and the first-order transfer constants, $H^{i}$, evaluated with the energy storing element at the port $i$ at its infinite value, as shown in Fig. 4. Note that transfer constants

\footnotetext{
${ }^{10}$ Unless all poles are decoupled, as defined in Section VI-B.

${ }^{11}$ From a notation perspective, we place the index(es) of the infinite valued element(s) in the superscript. An index 0 in the superscript (as in $R_{1}^{0}$ ) simply indicates that no reactive element is infinite valued, i.e., all elements are at their zero values.
}

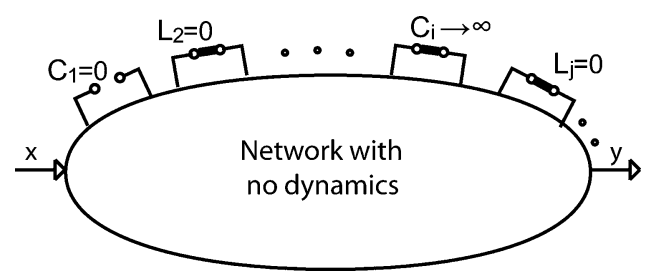

Fig. 4. Calculation of $\left.H^{i} \equiv(y / x)\right|_{C_{i} \rightarrow \infty ; e l s e: Z V}$ with $C_{i}$ shorted (infinite valued) and all other inductors and capacitors zero valued.

$H^{i}$ are easily evaluated using the low frequency calculations. The same line of argument can be applied for a combination of capacitors and inductors.

Note that the $\tau_{i}^{0}$ time constants have already been computed in determination of $b_{1}$, and, hence, all that needs to be calculated to determine $a_{1}$ are transfer constants, namely the $H^{i}$ coefficients. Also as we will see later, it is the ratios of $H^{i}$,s to $H^{0}$ that determine the zero location, and, hence, the exact details of $H^{i}$ 's do not matter to the extent we know how it is changed with respect to $H^{0}$, eliminating the need for recalculation of all parameters with a change in the circuit.

Equation (19) suggests that if all transfer constants of different orders are zero, there will be no zeros in the transfer function. This suggests an easy test to determine whether there is a zero in the transfer function by looking for capacitors shorting of which (or inductors opening of which) results in a nonzero low-frequency transfer function. ${ }^{12}$ We will see in Section V how this concept can be generalized to determine the number and location of the zeros. We will see in Section VI-D and Example VII-3 how (19) is used to include the effect of zeros in ZVT calculations. Next, we discuss the general case.

\section{Higher Order Terms: Generalized Time AND TRANSFER CONSTANTS (TTC)}

In this section, we generalize the approach to be able to determine the transfer function to any degree of accuracy (including exact result) by calculating higher-order $a_{n}$ and $b_{n}$ terms in (1). As we discussed earlier, the transfer function of the $N$ th-order system of Fig. 2 can be expressed in the form of (12). Note that in (12), the higher order sums are defined in such a way that for any two indexes $m$ and $n$ only one of the $\beta_{2}^{m n}$ and $\beta_{2}^{n m}$ is present in the sum to avoid multiple permutations of the same product. ${ }^{13}$ Since relabeling the capacitors should not change the poles and zeros of the transfer function, we conclude that $\beta_{2}^{m n}=\beta_{2}^{n m}$. A similar argument can be applied to the numerator to conclude that $\alpha_{2}^{m n}=\alpha_{2}^{n m}$. Also note that the higher order terms in (12) denoted by (...) have coefficients that are sums of products of at least three different capacitors.

We showed in Section IV that $b_{1}$ and $a_{1}$ in (1) are always given by (16) and (19), respectively, i.e., $\beta_{1}^{i}=R_{i}^{0}$ and $\alpha_{1}^{i}=$ $R_{i}^{0} H^{i}$ in (12). Now we determine higher order coefficients in

\footnotetext{
${ }^{12}$ Sometimes the transfer function has a pole that exactly coincides with a zero. When that happens the above procedure still predicts the existence of a zero, while there will be a pole at exactly the same location. An example is a parallel $R C$ network not connected to the rest of the circuit, which generates a at $1 / R C$ and a zero at exactly the same frequency.

${ }^{13}$ More generally, we can expect that any circular rotation of the $i j k \ldots$ indexes in the superscript of $\alpha_{l}^{i j k \ldots}$ and $\beta_{l}^{i j k \ldots}$ should result in the same value
} due to the same invariance to the labeling of the capacitors. 


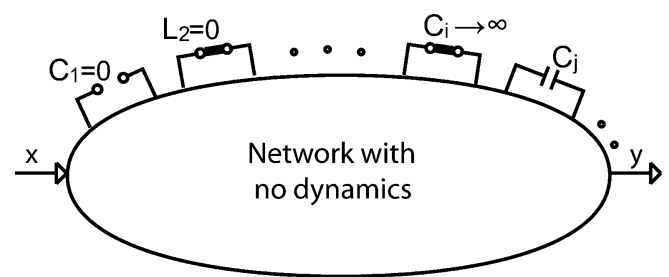

Fig. 5. The network with a single capacitor $C_{j}$ present, while $C_{i}$ is infinite valued (shorted) and all the inductors and capacitors zero valued.

(1). Next assume that we set $C_{i}$ to infinity and consider a capacitor $C_{j}$ at port $j$ while all other capacitors have a value of zero (i.e., are open). The network will look like Fig. 5. This is yet another first-order system different from the one in Fig. 3 used to determine $b_{1}$. The time constant of this new first-order system is

$$
\tau_{j}^{i}=R_{j}^{i} C_{j}
$$

where $R_{j}^{i}$ is the resistance seen at port $j$ with port $i$ infinite valued (capacitor $C_{i}$ shorted). Evaluating (12) with $C_{i} \rightarrow \infty$ and all other capacitors other than $C_{i}$ and $C_{j}$ at their zero value (i.e., open), we obtain

$$
\left.H(s)\right|_{C_{i} \rightarrow \infty}=\frac{C_{i} s \cdot\left(\alpha_{1}^{i}+\alpha_{2}^{i j} C_{j} s\right)}{C_{i} s \cdot\left(\beta_{1}^{i}+\beta_{2}^{i j} C_{j} s\right)}=\frac{\alpha_{1}^{i}}{\beta_{1}^{i}} \cdot \frac{1+\frac{\alpha_{2}^{i j}}{\alpha_{1}^{i}} C_{j} s}{1+\frac{\beta_{2}^{i j}}{\beta_{1}^{i}} C_{j} s}
$$

which is the transfer function of the new first-order system shown in Fig. 5. Equating the coefficient of $s$ in the denominator of (21) to (20), we obtain

$$
\beta_{2}^{i j}=\beta_{1}^{i} R_{j}^{i}=R_{i}^{0} R_{j}^{i}
$$

where we have used (15) in the last step. The second coefficient of the denominator, $b_{2}$, can be calculated as

$$
b_{2}=\sum_{i}^{1 \leqslant i} \sum_{j}^{<j \leqslant N} R_{i}^{0} C_{i} R_{j}^{i} C_{j}
$$

which, in general, can be written as

$$
b_{2}=\sum_{i}^{1 \leqslant i<j \leqslant N} \sum_{j}^{0} \tau_{i}^{0} \tau_{j}^{i}
$$

One important point is that since $\beta_{2}^{i j}=\beta_{2}^{j i}$, as discussed earlier, we conclude

$$
R_{i}^{0} R_{j}^{i}=R_{j}^{0} R_{i}^{j}
$$

This equality provides alternative ways of calculating higher order time constant products, some of which may be more straightforward to calculate in the actual circuit. Equivalently, we have the more useful form

$$
\tau_{i}^{0} \tau_{j}^{i}=\tau_{j}^{0} \tau_{i}^{j}
$$

Now to obtain $a_{2}$, we will let both $C_{i}$ and $C_{j}$ to go to infinity (short circuited) and all other reactive elements to be zero valued (e.g., open capacitors). The second-order input-output transfer constants are simply given by

$$
\left.H^{i j} \equiv H\right|_{\substack{C_{i}, C_{j} \rightarrow \infty \\ C_{k}=0 \\ i \neq j \neq k}}=\frac{\alpha_{2}^{i j}}{\beta_{2}^{i j}} .
$$

Since we have already determined $\beta_{2}^{i j}$ in (22), we determine that $\alpha_{2}^{i j}=R_{i}^{0} R_{j}^{i} H^{i j}$ and thus

$$
a_{2}=\sum_{i}^{1 \leqslant i} \sum_{j}^{<j \leqslant N} R_{i}^{0} C_{i} R_{j}^{i} C_{j} H^{i j}
$$

which again more generally can be written as

$$
a_{2}=\sum_{i}^{1 \leqslant i} \sum_{j}^{<j \leqslant N} \tau_{i}^{0} \tau_{j}^{i} H^{i j}
$$

where $H^{i j}$ is the low-frequency input-output transfer constant with both ports $i$ and $j$ shorted (or in general the reactive elements at ports $i$ and $j$ at their infinite value). The above approach can be continued by induction to determine higher order $a_{i}$ and $b_{i}$ coefficients using an inductive line of argument to (12).

In general, the $n$ th-order $b_{n}$ coefficient of the denominator is given by

$$
b_{n}=\sum_{i}^{1 \leqslant i<j<k} \sum_{j} \sum_{k \cdots}^{\leqslant \leqslant N} \ldots \tau_{i}^{0} \tau_{j}^{i} \tau_{k}^{i j} \ldots
$$

which is the same result as in [6], written in a more compact form.

The $a_{n}$ coefficient for the numerator is

$$
a_{n}=\sum_{i}^{1 \leqslant i<j<k} \sum_{j} \sum_{k \ldots}^{\leqslant N} \ldots \tau_{i}^{0} \tau_{j}^{i} \tau_{k}^{i j} \ldots H^{i j k \ldots}
$$

where $\tau_{k}^{i j \ldots}$ corresponds to the time constant due to the reactive element at port $k$ and the low frequency resistance seen at port $k$ when ports whose indexes are in the superscript $(i, j, \ldots)$ are infinite valued (shorted capacitors and opened inductors). In the presence of inductors a similar line of argument can be applied, noting that the time constant $\tau_{k}^{i j \ldots}$ associated with inductor $L_{k}$ is simply the inductance divided by $R_{k}^{i j \ldots}$ which is the resistance seen at port $k$ with the reactive elements at ports $i, j, \ldots$ at their infinite values. ${ }^{14}$ So, the time constants in (30) and (31) will have one of the following forms depending on whether there is an inductor or a capacitor connected to port $k$. For capacitor, $C_{i}$

$$
\tau_{i}^{j k \ldots}=C_{i} R_{i}^{j k \ldots}
$$

and for inductor, $L_{l}$

$$
\tau_{l}^{m n \cdots}=\frac{L_{l}}{R_{l}^{m n \ldots}} .
$$

${ }^{14}$ Equation (25) can be generalized noting the invariance of the $\beta_{l}^{i j k \cdots}$ to a rotation of the indexes to produce

$$
R_{i}^{0} R_{j}^{i} R_{k}^{i j} \ldots R_{m}^{i j k \cdots}=R_{j}^{0} R_{k}^{j} \ldots R_{m}^{j k l \ldots} R_{i}^{j k l \cdots} .
$$


Finally, $H^{i j k \ldots}$ is the $n$ th-order transfer constant evaluated with the energy storing elements at ports $i, j, k, \ldots$ at their infinite values (shorted capacitors and opened inductors) and all others zero valued (opened capacitors and shorted inductors). It is noteworthy that (30) indicates that the poles of the transfer function are independent of the definition of input and output and are only characteristics of the network itself, while the zeros are not a global property of the circuit and depend on the definition of the input and output ports and variables, as evident from the presence of the $H^{i j \cdots}$ terms. This is consistent with the fact that poles are the roots of the determinant of the $Y$ matrix [1] defined independent of the input and output ports.

Several observations are in order about this approach. First of all this approach is exact and makes it possible to determine the transfer function completely and exactly. More importantly, unlike writing nodal or mesh equations, one does not need to carry the analysis to its end to be able to obtain useful information about the circuit. Additional information about higher order poles and zeros can be obtained by carrying the analysis through enough steps to obtain the results to the desired level of accuracy. Also, the analysis is equally applicable to real and complex poles and zeros. Once mastered, this analysis method provides a fast and insightful means of evaluating transfer functions, as well as input and output impedances for general circuits. The generalized time and transfer constants (TTC) approach has several important and useful corollaries that will be discussed in the next section.

\section{COROLlaries AND APPLICATIONS}

\section{A. Number of Poles and Zeros}

It is a well known result that the number of poles (i.e., the number of natural frequencies) of a circuit is equal to the maximum number of independent initial conditions we can set for energy-storing elements. This result can also be easily deduced from (30), where the highest order nonzero $b_{n}$ is determined by the highest order nonzero time constant, $\tau_{i}^{j k \cdots}$ in the system.

It is easy to see that each purely capacitive loop with no other elements in the loop reduces the order of the system by one. This is because the highest order time constant associated with the last capacitor, when all the other ones are infinite valued (shorted) is zero, since the resistance seen by that capacitor in that case is zero ${ }^{15}$ (see Example VII-2 in Section VII). The same effect holds for an inductive cut-set, where only inductors are attached to a node. Again the time constant associated with the last inductor, when all others are infinite-valued (opened) is zero since the resistance seen is infinity.

The number of zeros can also be determined easily in the approach presented here. The number of zeros is determined by the order of the numerator polynomial, which is in turned determined by the highest order nonzero transfer constant, $H^{i j k \ldots, \text { in }}$

\footnotetext{
${ }^{15}$ In general, in a circuit with $N+1$ nodes (including ground), we can have up to $N(N+1) / 2$ distinct capacitors connected among the nodes. Despite this potentially large number of distinct capacitors, we can only define $N$ independent initial conditions. This can be seen if we assume that the voltages between individual capacitors from each node to ground is set. This sets all the node voltages, and, thus, no more initial condition can be defined independently, meaning we can have a maximum of $N$ poles in such a circuit.
}

(31). In other words, the number of zeros in the circuit is equal to the maximum number of energy-storing elements that can be $s i$ multaneously infinite-valued while producing a nonzero transfer constant $H^{i j k \ldots}$ from the input to the output. This way we can easily determine how many zeros there are in the transfer function of the system by inspection without having to write any equations (see Examples VII-2, VII-3, and VII-8). This is one of the advantages of this approach over that presented in [15].

\section{B. Decoupled Poles}

The second important corollary of the TTC relates to decoupled poles of the circuits. As we mentioned in Section IV-A, there is no one-to-one correspondence between the zero-valued time constants, $\tau_{i}^{0}$ and the poles' characteristic times $\left(-1 / p_{i}\right)$. However, an important exception is when a time-constant is decoupled from all other ones. This happens when a time constant does not change for any combination of shorting and opening of other energy-storing elements, i.e., in our notation

$$
\tau_{N}^{0}=\tau_{N}^{i}=\tau_{N}^{i j}=\cdots=\tau_{N}^{i j \ldots m} .
$$

In this case, the term $\left(1+\tau_{N}^{0} s\right)$ can be factored out of the denominator and the pole associated with it is simply a real one at $p_{N}=-1 / \tau_{N}^{0} \cdot{ }^{16}$

This concept can be generalized to a group or groups of time constants that can be decoupled from the rest of the time constants but internally coupled. An example is a multistage amplifier, with no interstage capacitors, where the time constants within each stage may be coupled and cannot be factored into products of first-order terms, however, it is possible to factor the numerator and denominator into product of lower order polynomials each associated with one set of externally uncoupled yet internally coupled set of time constants internal to each stage. This can be viewed as a partitioning of time constants into these mutually uncoupled subsets. (See Example VII-4).

$$
\begin{aligned}
& D(s)=1+b_{1} s+b_{2} s^{2}+\cdots \\
& =1+s \sum_{i=1}^{N} \tau_{i}^{0}+s^{2} \sum_{i}^{1 \leqslant i} \sum_{j}^{<j \leqslant N} \tau_{i}^{0} \tau_{j}^{i}+\cdots \\
& =1+s\left(\tau_{N}^{0}+\sum_{i=1}^{N-1} \tau_{i}^{0}\right) \\
& +s^{2}\left(\sum_{i=1}^{N-1} \tau_{i}^{0} \tau_{N}^{i}+\sum_{i}^{1 \leqslant i<j \leqslant N-1} \sum_{j}^{0} \tau_{i}^{0} \tau_{j}^{i}\right)+\cdots \\
& =\left(1+s \tau_{N}^{0}\right)+\left(1+s \tau_{N}^{0}\right) s \sum_{i=1}^{N-1} \tau_{i}^{0} \\
& +\left(1+s \tau_{N}^{0}\right) s^{2} \sum_{i}^{1 \leqslant i<j \leqslant N-1} \sum_{j}^{0} \tau_{i}^{i}+\cdots \\
& =\left(1+s \tau_{N}^{0}\right)\left[1+s \sum_{i=1}^{N-1} \tau_{i}^{0}\right. \\
& \left.+s^{2} \sum_{i}^{1 \leqslant i} \sum_{j}^{<j \leqslant N-1} \tau_{i}^{0} \tau_{j}^{i} \ldots\right]
\end{aligned}
$$

where the term in the bracket is of order of $s^{n-1}$. 


\section{Bandwidth Estimation Using ZVT's}

The $b_{1}$ coefficient calculated in (16) can be used to form a first-order estimate of $\omega_{h}$, the $-3 \mathrm{~dB}$ bandwidth of a circuit with a low-pass response. ${ }^{17}$ More importantly, it is a powerful design tool allowing the designer to identify the primary source of bandwidth limitation and can serve as a guide in making qualitative (e.g., topological) and quantitative (e.g., element values) changes to the circuit.

There are several simplifying assumptions involved in application of the basic ZVT method to bandwidth estimation. The original ZVT approach [5] assumes that there are no (dominant) zeros in the transfer function. Next in Section VI-D, we will augment the approach to account for dominant zeros in the transfer function and how to determine if they are present.

For now let us assume there are no dominant zeros in the transfer function. In this case, the transfer function can be approximated as

$$
H(s) \approx \frac{a_{0}}{1+b_{1} s+b_{2} s^{2}+\ldots+b_{n} s^{n}}
$$

which is the transfer function of low-pass system with a lowfrequency value of $a_{0}$.

At dc $(s=0)$, the only term in the denominator that matters is the leading 1 . As the frequency goes up and approaches $\omega_{h}$, the first term that becomes non-negligible would be $b_{1} s$, so in the vicinity of the $\omega_{h}$, (35) can be further approximated as a first-order system

$$
H(s) \approx \frac{a_{0}}{1+b_{1} s} .
$$

This implies that $\omega_{h}$, bandwidth of the complete system, can be approximated as [5]

$$
\omega_{h} \approx \frac{1}{b_{1}}=\frac{1}{\sum_{i=1}^{N} \tau_{i}^{0}}
$$

where $\tau_{i}^{0}$ are the zero value time constants defined by (14) and (17) for capacitors and inductors, respectively. 18 This approximation is conservative and underestimates the bandwidth [16].

As mentioned earlier, the coefficient $b_{1}$ is the sum of the pole characteristic times $\left(-1 / p_{i}\right)$ with no one-to-one corresponds among $p_{i}$ 's and $\tau_{i}$ 's, in general. Therefore, the imaginary parts of complex conjugate pole pairs cancel each other in the $b_{1}$ sum. As a result, ZVT method by itself does not provide any information about the imaginary part of the poles and is completely oblivious to it. This can result in gross underestimation of the bandwidth using (37), when the circuit has dominant complex

\footnotetext{
${ }^{17}$ As we saw in Section II, we can split a bandpass response with a welldefined mid-band gain into a low-pass and a high-pass one. We can arrive at the low pass response by setting certain biasing elements such as bypass capacitors, coupling capacitors, and RF chokes to their infinite values (shorted capacitor and open inductor). Then using the method of zero-value time constants we can approximate $\omega_{h}$. A dual process called the method of infinite-value time (IVT) constants discussed in Section VI-G can be use to estimate $\omega_{l}$ in the high-pass system.

${ }^{18}$ Intuitively, $\omega_{h}$ is the frequency at which the total output amplitude drops by a factor of $\sqrt{2}$ with respect to $a_{0}$. Under normal circumstances, at this point, the contribution of each one of the energy-storing elements is relatively small, and, hence, (37) can be thought of as the sum of their individual contributions to the gain reduction, assuming the other ones are not present.
}

poles which could lead to peaking in the frequency response. We will see how we can determine whether or not complex poles are present and how to estimate their quality factor $(Q)$ in Section VI-F and Example VII-5.

\section{Modified ZVT Bandwidth Estimation for a System With Zeros}

The ZVT approximation of (37) can be improved in the light of (19). In the presence of zeros using a similar argument used to arrive at (36), we conclude that close to $\omega_{h}$, the transfer function can be estimated as

$$
H(s) \approx a_{0} \cdot \frac{1+\frac{a_{1}}{a_{0}} s}{1+b_{1} s}
$$

which is a first-order system with a pole at $-1 / b_{1}$ and a zero at $-a_{0} / a_{1}$. The zero has the opposite effect on the magnitude of the transfer function compared to the pole since it increases the magnitude of the transfer function with frequency. According to (19), we have

$$
\frac{a_{1}}{a_{0}}=\sum_{i=1}^{N} \tau_{i}^{0} \frac{H^{i}}{H^{0}}
$$

First, let us assume that all $H^{i} / H^{0}$ terms are positive. In this case, the numerator's first-order coefficient, $a_{1} / a_{0}$, will be positive and the dominant zero is left-half plane (LHP). In this case, using the first Taylor series expansion terms of the numerator and the denominator, the $\omega_{h}$ estimate can be modified to

$$
\omega_{h} \approx \frac{1}{b_{1}-\frac{a_{1}}{a_{0}}}=\frac{1}{\sum_{i=1}^{N} \tau_{i}^{0}\left(1-\frac{H^{i}}{H^{0}}\right)} .
$$

If some of the $H^{i} / H^{0}$ terms are negative, it means that the transfer function has right-half plane (RHP) zeros. However, the RHP zeros have exactly the same effect on the amplitude as LHP ones unlike their phase response. Since $\omega_{h}$ only depends on the amplitude response and not the phase, a LHP zero at a given frequency should produce the exact same $\omega_{h}$ as a RHP zero at the same frequency. Therefore, in general, a better approximation for $\omega_{h}$ (assuming it exists) is

$$
\omega_{h} \approx \frac{1}{\sum_{i=1}^{N} \overline{\tau_{i}^{0}}}
$$

where

$$
\overline{\tau_{i}^{0}}=\tau_{i}^{0} \cdot\left(1-\left|\frac{H^{i}}{H^{0}}\right|\right)
$$

are modified ZVT's that are only different from the original ZVT's for reactive element which result in nonzero transfer constants when infinite valued (e.g., capacitors shorting of which does not make the gain zero). Also note that (41) and (42) subsume (40) for LHP zeros and reduces to (37) when there are no zeros, i.e., all $H^{i}$ terms are zero (corresponding to $a_{1}=0$ ). Usually only a few of the original ZVT's need be modified. Note that the correction to the time constants can be done at the same time 


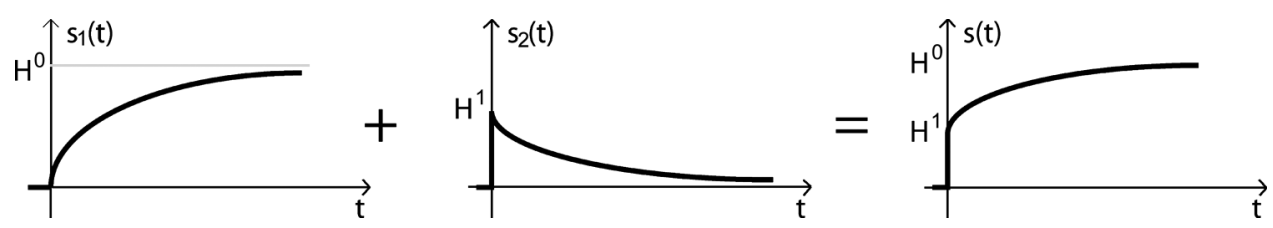

Fig. 6. Step response of a first-order system decomposed as the sum of the step response of a first-order low-pass system, $s_{1}(t)$ and high-pass systems, $s_{2}(t)$.

TABLE I

Relative Position of the Pole AND Zero in A First-Order System

\begin{tabular}{|l|l|l|}
\hline & $\frac{H^{0}}{H^{1}}>0$ & $\frac{H^{0}}{H^{1}}<0$ \\
\hline \hline$\left|\frac{H^{0}}{H^{1}}\right|<1$ & $|z|<|p|$ & $|z|<|p|$ \\
Same Half Plane & Opposite Half Plane \\
\hline$\left|\frac{H^{0}}{H^{1}}\right|>1$ & $\begin{array}{l}|z|>|p| \\
\text { Same Half Plane }\end{array}$ & $\begin{array}{l}|z|>|p| \\
\text { Opposite Half Plane }\end{array}$ \\
\hline
\end{tabular}

they are calculated simply by evaluating the change in the low frequency transfer function when the element is infinite valued.

Example VII-3 shows how the modified ZVT's produce a useful result in the presence of zeros, while regular ZVT's result is substantially inaccurate.

\section{E. Creation and Effect of Zeros}

Unlike poles that are natural frequencies of the circuit and, hence, are not affected by the choice of the input and output variables, zeros change with the choice of the input and output variables, as evident by the presence of $H^{i j k \cdots}$ terms in the $a_{n}$ 's. As mentioned earlier, as long as infinite valuing of some reactive elements results in nonzero low-frequency transfer function, there are zeros in the system.

1) Zeros in a First-Order System: For a first-order system with a single energy-storing element, we can easily obtain the following relation between the pole and the zero from (11)

$$
z=\frac{H^{0}}{H^{1}} \cdot p
$$

This expression is sufficient to evaluate the relative position of the zero with respect to the pole. It is clear from (43) that if the infinite- and zero-value transfer constants have opposite signs, the pole and the zero will be on two opposite half-planes. For instance, these correspond to low frequency gain of the system with a capacitor short- and open-circuited. In stable systems where the pole is in the LHP, the zero will be on the RHP for opposite polarities of $H^{0}$ and $H^{1}$, as in Example VII-2. On the other hand if $H^{0}$ and $H^{1}$ have the same polarity, the pole and the zero will be both on the LHP (see Example VII-3).

The magnitude of $H^{0} / H^{1}$ determines which one occurs at a lower frequency. As evident from (43), the zero happens first (at a lower frequency than the pole, i.e., $|z|<|p|$ ) when $\left|H^{0} / H^{1}\right|<1$. Alternatively, the pole occurs before the zero $(|p|<|z|)$, for $\left|H^{0} / H^{1}\right|>1$. This assessment can almost always be done by inspection because we only need to know the relative size and magnitude of $H^{0}$ and $H^{1}$, as summarized in Table I.

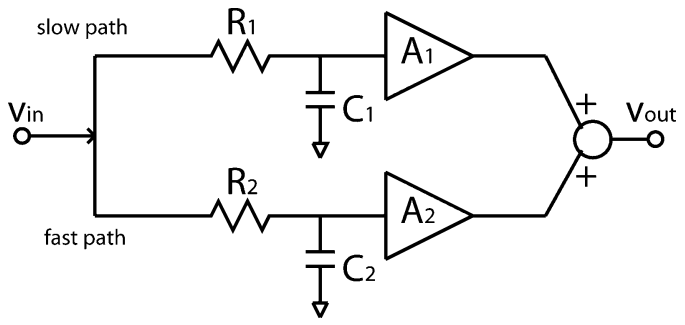

Fig. 7. System consisting of two signal paths each with a first-order response followed by unilateral ideal voltage amplifiers.

2) Time-Domain Response Due to a Zero: In a first-order system, the transfer function of (11) can be expressed as the sum of a first-order low-pass and a first-order high-pass system, i.e.,

$$
H(s)=\frac{H^{0}}{1+\tau s}+\frac{H^{1}}{1+\frac{1}{\tau s}}
$$

resulting in a step response of

$$
s(t)=H^{0}\left(1-e^{-t / \tau}\right) u(t)+H^{1} e^{-t / \tau} u(t)
$$

where $u(t)$ is the unit step. Since both responses have the same time constant, $\tau$, the overall response would be an exponential with an initial value, $H^{1}$, and a final value, $H^{0}$, and a time constant, $\tau$, as shown in Fig. 6. Again, the relative size and polarities of $H^{0}$ and $H^{1}$ determines the general behavior of the response.

When $H^{0}$ and $H^{1}$ have opposite polarities, the low- and highpass responses will go in different directions resulting in an undershoot. On the other hand, when $H^{0}$ and $H^{1}$ have the same polarities, but $0<H^{0} / H^{1}<1$, the step response's initial value $\left(H^{1}\right)$ is greater than its final value $\left(H^{0}\right)$, and, hence, there will be an overshoot. For $1<H^{0} / H^{1}$, the step response starts at the smaller value $H^{1}$ at $t=0$ and then increases exponentially to its final value (similar to Fig. 6.)

Next, let us consider a second-order system with two arbitrary real poles and a single zero. The transfer function of such a system can be written as

$$
H(s)=H^{0} \cdot \frac{1-\frac{s}{z}}{\left(1-\frac{s}{p_{1}}\right)\left(1-\frac{s}{p_{2}}\right)}=H^{0} \cdot \frac{1+\tau_{z} s}{\left(1+\tau_{1} s\right)\left(1+\tau_{2} s\right)}
$$

where $H^{0}$ is the zero-value transfer constant of the circuit. We can assume $\left|p_{1}\right|<\left|p_{2}\right|$ (i.e., $\tau_{1}>\tau_{2}$ ) without loss of generality. The zero can be LHP or RHP and $H(s)$ can be written, as the sum of partial fractions, as depicted in Fig. 7 [17]. $A_{1}$ and $A_{2}$ are given by

$$
\begin{aligned}
& A_{1}=H^{0} \cdot \frac{p_{2}}{z} \cdot \frac{z-p_{1}}{p_{2}-p_{1}} \\
& A_{2}=-H^{0} \cdot \frac{p_{1}}{z} \cdot \frac{z-p_{2}}{p_{2}-p_{1}}
\end{aligned}
$$




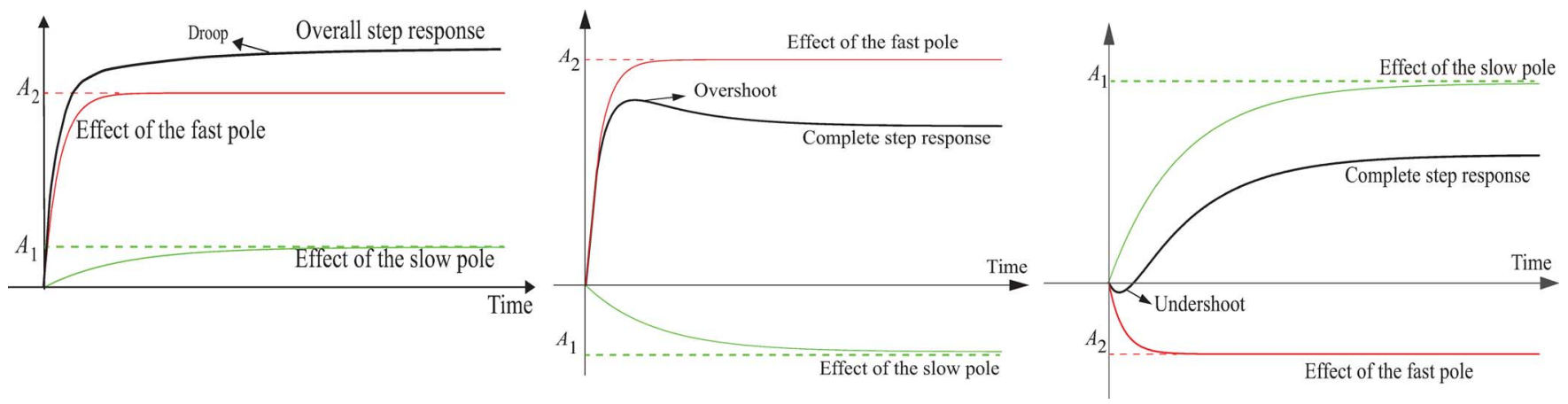

Fig. 8. Step responses of two paths with a) same polarities $\left(A_{1} A_{2}>\right) 0$ (droop); b) opposite polarities $\left(A_{1} A_{2}<0\right)$ and $\left|A_{2}\right|>\left|A_{1}\right|$ (overshoot); c) opposite polarities $\left(A_{1} A_{2}<0\right)$ and $\left|A_{2}\right|<\left|A_{1}\right|$ (undershoot).

$$
H(s)=\frac{A_{1}}{1-\frac{s}{p_{1}}}+\frac{A_{2}}{1-\frac{s}{p_{2}}}=\frac{A_{1}}{1+\tau_{1} s}+\frac{A_{2}}{1+\tau_{2} s}
$$

where $\tau_{1}=-1 / p_{1}$ and $\tau_{2}=-1 / p_{2}$ are the pole characteristic times $\left(\tau_{1}>\tau_{2}\right)$ and we have

$$
\tau_{z}=-\frac{1}{z}=\frac{A_{2} \tau_{1}+A_{1} \tau_{2}}{A_{1}+A_{2}} .
$$

This system can be completely modeled using the dual-path system of Fig. 7, where the upper path with the low-frequency gain $A_{1}$ is slower than the lower one with the gain $A_{2}$. It has two first-order parallel paths each with a single pole transfer function and no zeros. While the two-path system of Fig. 7 may appear quite idealized, it can be used to represent any second-order system with two LHP real poles and a zero. Each of these poles would be at exactly the same frequency as those of the real poles of the original second-order system. Interestingly, a new real zero is created that did not exist in either of the constitutive first-order systems of Fig. 7. This is essentially due to the fact that the summation of the two different responses in the two parallel systems will result in the two responses canceling each other at a complex frequency.

Using the decomposition of Fig. 7, we conclude that the timedomain response is the sum of the responses of the two firstorder single-pole systems, i.e.,

$$
s(t)=A_{1}\left(1-e^{-t / \tau_{1}}\right)+A_{2}\left(1-e^{-t / \tau_{2}}\right) .
$$

A careful look at (48) indicates that if the gains of the two paths have the same polarities (i.e., $A_{1} A_{2}>0$ ), the zero is LHP and falls between the two poles, as (48) could be looked at as the weighted average of $\tau_{1}$ and $\tau_{2}$. In this case, the response is the sum of two exponentials going in the same direction with two different time constants as shown in Fig. 8(a), resulting in a droop, as the response associated with $p_{2}$ settles quickly but the response corresponding to $p_{1}$ takes a while longer to reach its final value.

The condition $-1<A_{1} / A_{2}<0$ results in a LHP zero closer to the origin than either $p_{1}$ and $p_{2}$. Again using the equivalent system of Fig. 7, we see that the two paths have opposite polarities and the magnitude of the slower path's gain $\left(A_{1}\right)$ is smaller than the faster path $\left(A_{2}\right)$, as shown in Fig. 8(b). The faster path which has a higher gain results in an overshoot in the response

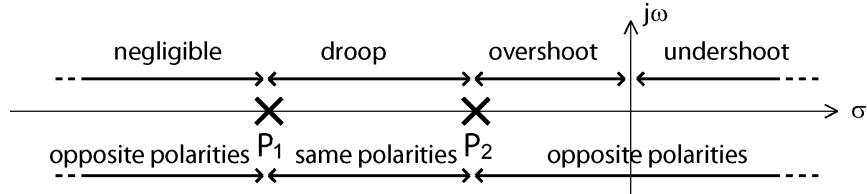

Fig. 9. Impact of the location of the zero on the step response behavior and the implied relative polarity of the paths in its two-path equivalent.

that is eventually reduced by the slower path. Note that this overshoot is not caused by complex poles, rather by the zero in the transfer function.

We have a LHP zero if $A_{1} / A_{2}<-\tau_{1} / \tau_{2}$, but the zero is at a higher frequency than either $p_{1}$ and $p_{2}$ and, hence, usually has a negligible effect. Another way to see this is by noting that in this case slow response has significantly higher gain than the faster one, so it modifies the slope of the response of the primary path slightly but its effect is completely diminished by the time the high-gain slower path reaches steady-state.

When the gains have opposite polarities and their ratio is in the range $-\tau_{1} / \tau_{2}<A_{1} / A_{2}<-1$, we have a RHP zero. The slower path still has a larger gain magnitude but the faster one has high enough gain to produce an undershoot, as in Fig. 8(c). The undershoot is a trait associated with RHP zeros.

To summarize, two parallel paths with the same polarity result in a real zero between $p_{1}$ and $p_{2}$ which causes a droop in the step response. Having two signal paths with opposite polarities can result in an undershoot if the faster path has a smaller gain and an overshoot if it has a larger gain. These results are summarized in Fig. 9.

As a side note, although in the dual path system of Fig. 7 there are two capacitors, $C_{1}$ and $C_{2}$, shorting of either one results in a nonzero transfer function. Nonetheless, there is only one zero in the transfer function, since simultaneous shorting (infinitevaluing) of both results in a zero transfer function $\left(H^{12}=0\right)$, as given by the criterion discussed in Section VI-A.

\section{F. Properties of Second-Order Systems}

The transfer function of a second-order system can be expressed in terms of the natural frequency, $\omega_{n}$, and the quality factor, $Q$

$$
H(s)=\frac{N(s)}{1+\frac{s}{Q \omega_{n}}+\frac{s^{2}}{\omega_{n}^{2}}}
$$


where $N(s)$ is the numerator and $Q=1 / 2 \zeta$ is a measure of the energy loss per cycle in the system $(\zeta$ is called the damping ratio). Note that the quality factor and the damping factor are defined independent of the numerator. Evaluating (50) in terms of $b_{n}$ coefficients, we obtain

$$
Q=\frac{1}{2 \zeta}=\frac{\sqrt{b_{2}}}{b_{1}}
$$

which for a second-order system can be written in terms of the time-constants

$$
\frac{1}{Q}=2 \zeta=\frac{\tau_{1}^{0}+\tau_{2}^{0}}{\sqrt{\tau_{1}^{0} \tau_{2}^{1}}}=\sqrt{\frac{\tau_{1}^{0}}{\tau_{2}^{1}}}+\sqrt{\frac{\tau_{2}^{0}}{\tau_{1}^{2}}}
$$

where (26) has been used in the last step to arrive at a more symmetrical result. It is easy to see from quadratic roots of the denominator of (50) that for $Q>(1 / 2)$ the roots of the denominator become complex.

The undamped resonance or natural frequency, $\omega_{n}$, can be readily related to the $b_{2}$ by

$$
\omega_{n}=\frac{1}{\sqrt{b 2}}
$$

which can be written in terms of the time constants as

$$
\omega_{n}=\frac{1}{\sqrt{\tau_{1}^{0} \tau_{2}^{1}}}=\frac{1}{\sqrt{\tau_{2}^{0} \tau_{1}^{2}}}
$$

Equations (51) and (53) are useful in the light of the relatively straightforward relation between $Q$ and $\omega_{n}$ with $b_{1}$ and $b_{2}$ coefficients given by (51) (see Example VII-5). They are also useful as approximations in higher order to estimate the amplitude and the frequency of peaking of the response (see Example VII-5).

\section{G. Infinite Value Time Constants}

We saw earlier in Section II ( ${ }^{3}$ ) that the transfer function of a bandpass system with a well-defined pass-band can be factored into the part responsible for the low-frequency behavior in terms of inverse poles and zeros, which results in a high pass response and a part responsible for the high-frequency behavior in terms of conventional poles and zeroes that form a low pass response. We can apply the infinite value time-constant (IVT) approach to determine the low-frequency behavior, in particular, its low $-3-\mathrm{dB}$ frequency, $\omega_{l}$.

To have a unity response at high frequencies in a high-pass response, the numerator should be of the same order as the denominator. If there are no zeros close to $\omega_{l}$, we have

$$
\begin{aligned}
H(s) & \approx \frac{a_{n} s^{n}}{1+b_{1} s+b_{2} s^{2}+\ldots+b_{n} s^{n}} \\
& =\frac{a_{m i d}}{1+\frac{b_{n-1}}{b_{n} s}+\ldots+\frac{1}{b_{n} s^{n}}}
\end{aligned}
$$
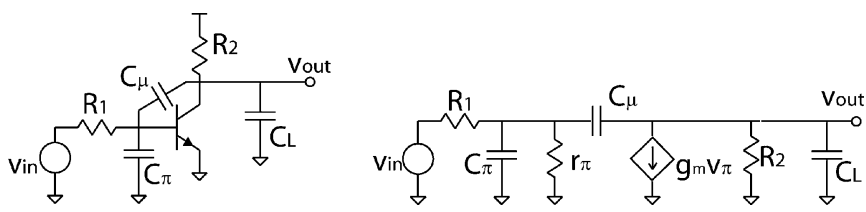

Fig. 10. a) Common-emitter stage with capacitors $C_{\pi}$ and $C \mu$ driving a load capacitor, $C_{L} ;$ b) its small-signal equivalent assuming $r_{o}$ is large (or absorbed into $R_{2}$ ).

where $a_{m i d}=a_{n} / b_{n}$ is the gain at very high frequencies. As we lower the frequency, the most dominant term affecting $\omega_{l}$ is $b_{n-1} / b_{n}$.

For an $n$ th-order high-pass system, we can approximate $\omega_{l}$ with $b_{n-1} / b_{n}$, i.e.,

$$
\begin{aligned}
\omega_{l} & \approx \frac{b_{n-1}}{b_{n}}=\frac{\sum_{i}^{1 \leqslant i<j<k} \sum_{j} \sum_{k \ldots N}^{\leqslant N} \ldots \tau_{i}^{0} \tau_{j}^{i} \tau_{k}^{i j} \ldots}{\tau_{i}^{0} \tau_{j}^{i} \tau_{k}^{i j} \ldots \tau_{n}^{i j \ldots m}} \\
& =\frac{1}{\tau_{1}^{23 \ldots n}}+\frac{1}{\tau_{2}^{13 \ldots n}}+\ldots+\frac{1}{\tau_{n}^{12 \ldots(n-1)}}
\end{aligned}
$$

where we have used the rotational symmetry discussed in the 14 . The time constant, $\tau_{i}^{12 \ldots(i-1)(i+1) \ldots n}$, which we will denote as, $\tau_{i}^{\infty}$, is the time constant for the $i$ th element with all other ports at infinite values, hence called an infinite-value time constant, IVT. 19

This can be summarized as

$$
\omega_{l} \approx \frac{b_{n-1}}{b_{n}}=\sum_{i=1}^{N} \frac{1}{\tau_{i}^{\infty}}
$$

where

$$
\tau_{i}^{\infty}=C_{i} R_{i}^{\infty}
$$

for capacitor, $C_{i}$, and

$$
\tau_{l}^{\infty}=\frac{L_{l}}{R_{l}^{\infty}}
$$

for inductor $L_{l}$. Resistance $R_{i}^{\infty}$ is the resistance seen looking into port $i$ when the capacitors and inductors at all other ports are at their infinite values (shorted capacitors and opened inductors).

\section{EXAMPLES}

In this section, we present several examples of the application of the TTC method. We use well-known circuits to demonstrate application of the method in a familiar context.

1) Common-Emitter, ZVT's: Consider the common-emitter stage of Fig. 10(a) with three capacitors, $C_{\pi}, C_{\mu}$, and $C_{L}$ connected at the output. The equivalent small-signal model for

\footnotetext{
${ }^{19}$ When the energy-storing elements are capacitors only, this method is often referred to as the method of short-circuit time constants. Obviously, the term infinite value time-constant is advantageous because it applies to both capacitors and inductors.
} 


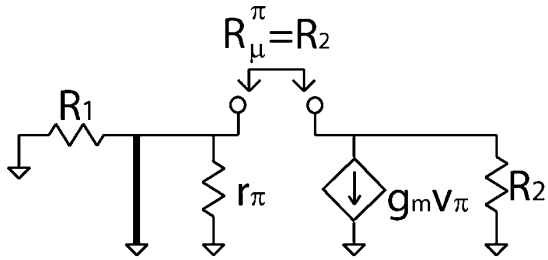

a)

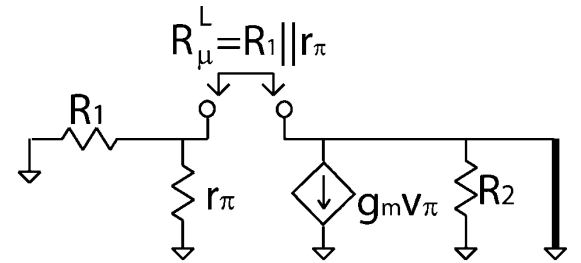

b)

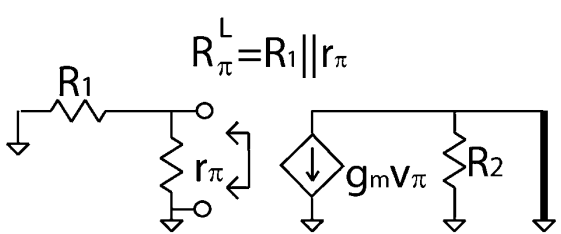

c)

Fig. 11. Equivalent circuit used to calculate for the common-emitter stage of Fig. 10: a) $\tau_{\mu}^{\pi}$, b) $\tau_{\mu}^{L}$, c) $\tau_{\pi}^{L}$.

this stage is shown in Fig. 10(b). The low-frequency gain is obviously

$$
a_{0}=H^{0}=\frac{v_{\text {out }}}{v_{1}} \cdot \frac{v_{1}}{v_{\text {in }}}=-g_{m} R_{2} \cdot \frac{r_{\pi}}{r_{\pi}+R_{1}} .
$$

First let us calculate the coefficient $b_{1}$ by calculating the three ZVT's associated with capacitors. In this example, we will use the $\pi, \mu$, and $L$ indexes to identify the elements. To determine the zero-value resistance seen by $C_{\pi}$, we null (short-circuit) the input voltage source and by inspection, we have 20

$$
\tau_{\pi}^{0}=C_{\pi} R_{\pi}^{0}=C_{\pi}\left(R_{1} \| r_{\pi}\right)
$$

The resistance seen by $C_{\mu}$ we have

$$
\tau_{\mu}^{0}=C_{\mu} R_{\mu}^{0}=C_{\mu}\left[R_{1} \| r_{\pi}+R_{2}+g_{m}\left(R_{1} \| r_{\pi}\right) R_{2}\right]
$$

and the zero-value resistance seen by $C_{L}$ is trivial as nulling the $v_{i}$ sets the dependent current source to zero (open circuit) and hence

$$
\tau_{L}^{0}=C_{L} R_{L}^{0}=C_{L} R_{2}
$$

${ }^{20} \mathrm{~A}$ useful result in many of these calculations is the resistance seen by capacitors connected between various terminals of a three terminal transistor with external resistors, $R_{B}, R_{C}$, and $R_{E}$ from the base (gate), collector (drain), and emitter (source) to ac ground respectively. It can be shown that ignoring transistor's intrinsic output resistance, $r_{o}$, the base-emitter (or gate-source) resistance, $R_{\pi}^{0}$, is given by

$$
R_{\pi}^{0}=r_{\pi} \| \frac{R_{B}+R_{E}}{1+g_{m} R_{E}}
$$

The base-collector (or gate-drain) resistance, $R_{\mu}^{0}$ is given by

$$
R_{\mu}^{0}=R_{\text {left }}+R_{\text {right }}+G_{m} R_{\text {left }} R_{\text {right }}
$$

where

$$
\begin{aligned}
R_{\text {left }} & \equiv R_{B} \|\left[r_{\pi}+(1+\beta) R_{E}\right] \\
R_{\text {right }} & \equiv R_{C} \\
G_{m} & \equiv \frac{1}{r_{m}+R_{E}}=\frac{g_{m}}{1+g_{m} R_{E}} .
\end{aligned}
$$

Note that $R_{\text {left }}$ it the resistance seen between the base (gate) and the ac ground which reduces to $R_{B}$ for a MOSFET $(\beta \rightarrow \infty)$. Resistance $R_{\text {right }}$ is the resistance between the collector (drain) and ac ground, and finally $G_{m}$ is the effective trans-conductance. The resistance seen between the collector and the emitter (drain and source), $R_{\theta}^{0}$, is given by

$$
R_{\theta}^{0} \approx \frac{R_{C}+R_{E}}{1+g_{m} R_{E}}
$$

where the approximation disappears for $\beta \rightarrow \infty$. Note that $R_{\theta}^{0}$ is not the same as the resistance seen between the collector and ground, namely, $R_{\text {right }}$.
Applying (16), we obtain

$$
b_{1}=\sum_{i} \tau_{i}^{0}=\tau_{\pi}^{0}+\tau_{\mu}^{0}+\tau_{L}^{0}
$$

In a numerical example 21 we have $H^{0}=-57$ and the time constants are $\tau_{\pi}^{0} \approx 70 \mathrm{ps}, \tau_{\mu}^{0} \approx 1,200 \mathrm{ps}$, and $\tau_{L}^{0}=400 \mathrm{ps}$ leading to a bandwidth estimate of $\omega_{h} \approx 1 / b_{1} \approx 2 \pi \cdot 95 \mathrm{MHz}$. A SPICE simulation predicts a $-3 \mathrm{~dB}$ bandwidth of $f_{h}=97 \mathrm{MHz}$ in close agreement with the above result.

2) Common-Emitter, Exact Transfer Function: The common emitter stage of Fig. 10 has three capacitors, but in fact we can only set $t w o$ independent initial conditions because of the capacitive loop, i.e., it has only two independent degrees of freedom.

We have already determined the coefficient $b_{1}$ in (60). Now let us determine $b_{2}$ using (24). To do so, we determine three time constants by short-circuiting the associated element with the superscript and looking at the impedance seen by the elements designated by the subscripts. Unlike ZVT's, all of which we needed, there are six such combinations of these time constants $\left(\tau_{\mu}^{\pi}, \tau_{\mu}^{L}, \tau_{\pi}^{L}, \tau_{\pi}^{\mu}, \tau_{L}^{\mu}\right.$, and $\left.\tau_{L}^{\pi}\right)$, out of which we can pick any three to cover each two-way combination once and only once to be coupled with the ZVT's. There are many combinations, but noting that the expression for $\tau_{\mu}^{0}$ is longer than other ZVT's, we try to pick the ones that avoid it to make our calculation more straightforward, i.e.,

$$
\begin{aligned}
\tau_{\mu}^{\pi} & =C_{\mu} R_{2} \\
\tau_{\pi}^{L} & =C_{\pi}\left(r_{\pi} \| R_{1}\right) \\
\tau_{\mu}^{L} & =C_{\mu}\left(r_{\pi} \| R_{1}\right)
\end{aligned}
$$

that are calculated using the circuits shown in Fig. 11. These combined with the ZVT's calculated in (60) produce

$$
\begin{aligned}
b_{2} & =\sum_{i}^{1 \leqslant i} \sum_{j}^{<j \leqslant 3} \tau_{i}^{0} \tau_{j}^{i} \\
& =\tau_{L}^{0} \tau_{\pi}^{L}+\tau_{\pi}^{0} \tau_{\mu}^{\pi}+\tau_{L}^{0} \tau_{\mu}^{L} \\
& =\left(r_{\pi} \| R_{1}\right) R_{2} \cdot\left(C_{\pi} C_{\mu}+C_{\pi} C_{L}+C_{\mu} C_{L}\right) .
\end{aligned}
$$

${ }^{21} \mathrm{We}$ assume the following parameters: a collector current of $1 \mathrm{~mA}$ (translating to $g_{m}=40 \mathrm{mS}$ ), $\beta_{0}=100, C_{j e}=20 \mathrm{fF}, C_{j c}=20 \mathrm{fF}, C_{j s}=50 \mathrm{fF}$, and $\tau_{F}=2$ ps which corresponds to a $C_{b}=g_{m} \tau_{F}=80 \mathrm{fF} / \mathrm{mA}$ at room temperature, leading to $C_{\pi}=C_{j e}+C_{b}=100 \mathrm{fF}$ and $C_{\mu}=C_{j c}=20 \mathrm{fF}$. Now consider an external capacitor on the output of $C_{\text {out }}=150 \mathrm{fF}$ which together with $C_{j s}$ form $C_{L}=C_{\text {out }}+C_{j s}=200 \mathrm{fF}$. These values correspond to a transistor cut-off frequency, $f_{T} \approx 53 \mathrm{GHz}$. We also assume $R_{1}=1 \mathrm{k} \Omega$ and $R_{2}=2 \mathrm{k} \Omega$ in the circuit of Fig. 10 . 


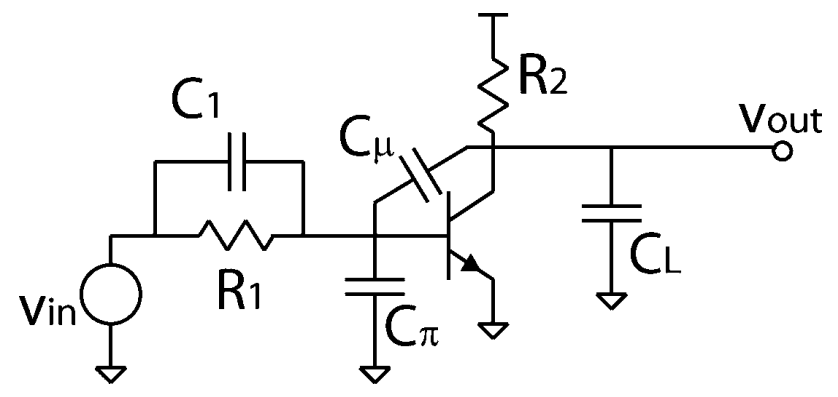

Fig. 12. Common-emitter stage with a capacitor $C_{1}$ in parallel with the input resistance $R_{1}$.

From (30), we see that with three energy-storing elements, $b_{3}=$ $\tau_{1}^{0} \tau_{2}^{1} \tau_{3}^{12}$ which has to be zero since $\tau_{3}^{12}=0$ due to the capacitive loop. Thus, the system is only second-order with two poles as expected.

Since $H^{\pi}$ and $H^{L}$ are zero, we only need to calculate $H^{\mu}$. Shorting $C_{\mu}$, the circuit reduced to a resistive divider between $R_{1}$ and $\alpha r_{m} \| R_{2}$. The voltage gain is simply given by the resistive divider ratio, i.e.,

$$
H^{\mu}=\frac{\alpha r_{m} \| R_{2}}{R_{1}+\alpha r_{m} \| R_{2}}=\frac{r_{\pi}}{r_{\pi}+R_{1}} \cdot \frac{R_{2}}{R_{\mu}^{0}}
$$

Hence, the voltage transfer function can be determined from (11)

$$
H(s)=H^{0} \cdot \frac{1+\frac{H^{\mu}}{H^{0}} \tau s}{1+b_{1} s+b_{2} s^{2}}=H^{0} \cdot \frac{1-\frac{C_{\mu}}{g_{m}} s}{1+b_{1} s+b_{2} s^{2}}
$$

where the $b_{1}$ and $b_{2}$ were calculated (60) and (61), respectively. It is noteworthy that in this example, $H^{0}$ and $H^{\mu}$ have opposite signs, which results in a RHP zero, $z=g_{m} / C_{\mu}$, in the transfer function as expected. Note the relative ease of calculation of this transfer function compared to writing the nodal equations.

3) Common-Emitter, Input Zero: Let us consider the common emitter stage of previous examples where a capacitor $C_{1}$ is introduced in parallel with $R_{1}$ at the input, as shown in Fig. 12. The time constants calculated in Example VII-1 remain the same. Only a new time constant, $\tau_{1}^{0}$, associated with $C_{1}$ will appear in $b_{1}$, which is easily calculated to be

$$
\tau_{1}^{0}=C_{1}\left(R_{1} \| r_{\pi}\right)
$$

Applying (16) to estimate the bandwidth, the ZVT simply predicts a smaller $\omega_{h}$ than when $C_{1}$ is not there since we have just added a new, and potentially large time constant to the $b_{1}$ sum.

Numerically, with $C_{1}=4.3 \mathrm{pF}$ and all other values the same as those in Example VII-1, we have $\tau_{1}^{0} \approx 3.07 \mathrm{~ns}$ and the bandwidth estimate according to the conventional ZVT given in (16) is $\omega_{h} \approx 2 \pi \cdot 34 \mathrm{MHz}$. However, this time SPICE predicts a $-3 \mathrm{~dB}$ bandwidth of $f_{h}=482 \mathrm{MHz}$ which is more than an order of magnitude higher! The reason is that $C_{1}$ introduces a LHP zero since shorting it results in a nonzero transfer function $H^{1}$ with the same polarity as $H^{0}$. In this example, the frequency of this zero has been adjusted by choosing the right value of $C_{1}$ to coincide with the first pole of the transfer function effectively canceling it.

In this example, although (16) is still providing a conservative value, it is too far off to be of much use. The basic premise for the approximation in the conventional ZVT is the absence of any zeros close to or below $\omega_{h}$. Once this assumption is violated, the conventional ZVT does not provide much useful information.

This problem can be remedied by using the modified ZVT's, as defined in (42). To determine which time-constant must be modified, we calculate the low-frequency transfer functions

$$
\begin{aligned}
H^{\pi} & =0 \\
H^{\mu} & =\frac{\alpha r_{m} \| R_{2}}{R_{1}+\alpha r_{m} \| R_{2}} \approx \frac{r_{m}}{R_{1}} \\
H^{L} & =0 \\
H^{1} & =-g_{m} R_{2} .
\end{aligned}
$$

Determination of $H^{1}$ (which is the only $H$ coefficient with a significant value in this case) is straightforward, as it is simply the gain without the input voltage divider. Since $H^{\mu}$ and $H^{1}$ are nonzero, the two ZVT's that need to be modified are

$$
\begin{aligned}
& \overline{\tau_{\mu}^{0}}=\tau_{\mu}^{0} \cdot\left(1-\left|\frac{H^{\mu}}{H^{0}}\right|\right)=\tau_{\mu}^{0} \cdot(1-0.0004) \approx \tau_{\mu}^{0} \\
& \overline{\tau_{1}^{0}}=\tau_{1}^{0} \cdot\left(1-\left|\frac{H^{1}}{H^{0}}\right|\right)=\tau_{1}^{0} \cdot(1-1.4)=-0.4 \cdot \tau_{1}^{0} .
\end{aligned}
$$

As can be seen, the modification to $\overline{\tau_{\mu}^{0}}$ is negligible, while the modified $\overline{\tau_{1}^{0}}$ has a significant impact.

The new bandwidth estimate using the effective time constants in (41) is $\omega_{h} \approx 1 /(70 \mathrm{ps}+1.2 \mathrm{~ns}+400 \mathrm{ps}-1.23 \mathrm{ps}) \approx$ $2 \pi \cdot 362 \mathrm{MHz}$ which is much closer to the SPICE results of $f_{h}=482 \mathrm{MHz}$ than the estimate of $34 \mathrm{MHz}$ obtained from the conventional ZVT's. As we can see after the correction, it is the time constants associated with $C_{\mu}$ and $C_{L}$ in conjunction with $R_{2}$ that become significant and determine the bandwidth. This result can be further improved by calculating coefficient $b_{2}$ using (24).

One thing to note is that we can quickly verify whether we need to use the approximation of (40), or (37) simply suffices, by determining if setting any of the energy-storing elements to its infinite value results in a nonzero transfer function, namely if we have any nonzero $H^{i}$ terms. For nonzero $H^{i}$ we should evaluate $\left|\tau_{i}^{0} H^{i} / H^{0}\right|$ and see if its inclusion has a considerable effect on $b_{1}$. If that is the case, it should be subtracted from $b_{1}$ and otherwise simply ignored.

4) Cascode Stage: The cascode stage is illustrated in Fig. 13(a) with its small signal equivalent circuit in Fig. 13(b). We note that for a large transistor output resistance, $r_{o}$, the time constants associated with $C_{\pi 1}, C_{\mu 1}, C_{c 1}$, and $C_{\pi 2}$ are decoupled from those of $C_{\mu 2}, C_{c 2}$, and $C_{o}$, since the time-constant of one group remains unaffected by any combination of shorting and opening of the other batch. A closer look also indicates that capacitors $C_{c 1}=C_{j s}$ and $C_{\pi 2}$ are in parallel, so are $C_{c 2}=C_{j s}, C_{\mu 2}$, and $C_{o}$, and, hence, we define $C_{e}=C_{c 1}+C_{\pi 2}$ and $C_{L}=C_{c 2}+C_{\mu 2}+C_{o}$, and deal with four capacitors from this point on. 

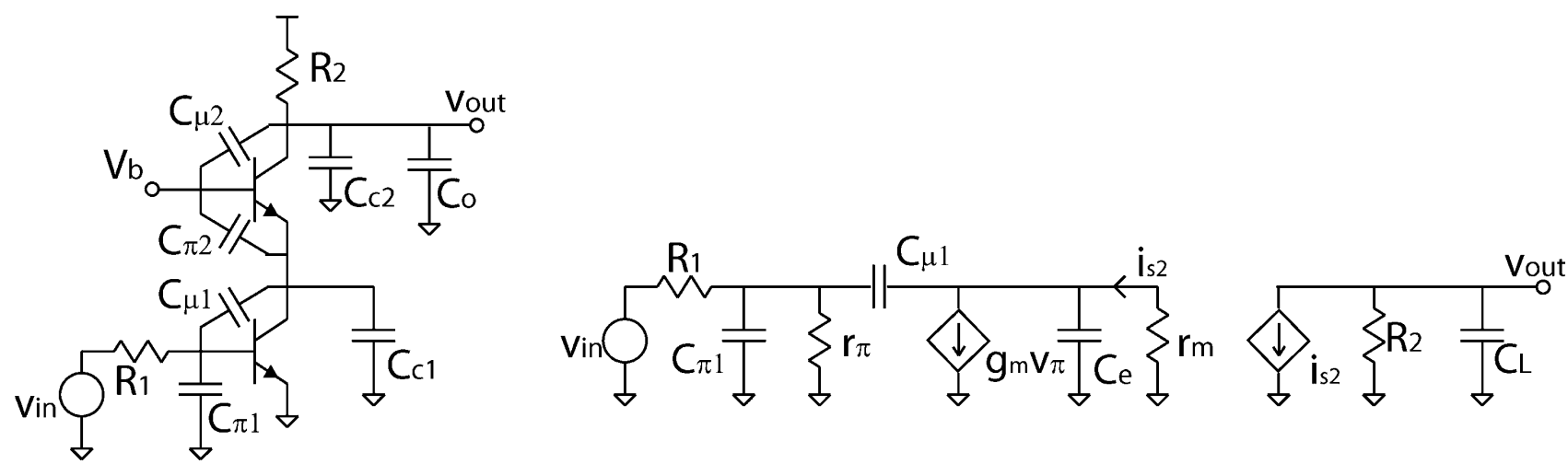

Fig. 13. a) Cascode stage driving a load capacitor, $C_{L}$; b) its approximate small-signal equivalent circuit assuming large $r_{o}$.

The four ZVT time constants for these capacitors are

$$
\begin{aligned}
\tau_{\pi}^{0} & =C_{\pi}\left(R_{1} \| r_{\pi}\right) \\
\tau_{\mu}^{0} & =C_{\mu}\left[R_{1} \| r_{\pi}+\alpha r_{m}+g_{m}\left(R_{1} \| r_{\pi}\right) \alpha r_{m}\right] \\
& \approx C_{\mu}\left[2\left(R_{1} \| r_{\pi}\right)+r_{m}\right] \\
\tau_{e}^{0} & =C_{e} \alpha r_{m} \\
\tau_{L}^{0} & =C_{L} R_{2} .
\end{aligned}
$$

We notice that $\tau_{\mu}^{0}$ which was the dominant source bandwidth reduction in the common emitter of Example VII-1, is now reduced significantly, which explains the well-known advantage of the cascode. A numerical calculation of the ZVT's with the same values as the previous examples, predicts $\omega_{h} \approx 2 \pi$. $294 \mathrm{MHz}$, where SPICE simulations indicate a $-3 \mathrm{~dB}$ frequency of $f_{h}=337 \mathrm{MHz}$.

Noting that $\tau_{L}$ is decoupled from the other time constants, we can express the transfer function as

$$
H(s)=H^{0} \cdot \frac{1+\frac{a_{1}^{\prime}}{a_{0}} s}{\left(1+b_{1}^{\prime} s+b_{2}^{\prime} s^{2}\right)} \cdot \frac{1}{1+\tau_{L} s}
$$

where

$$
\begin{aligned}
& b_{1}^{\prime}=\tau_{\pi}^{0}+\tau_{\mu}^{0}+\tau_{e}^{0} \\
& b_{2}^{\prime}=\alpha r_{m}\left(R_{1} \| r_{\pi}\right)\left(C_{\pi} C_{\mu}+C_{\pi} C_{e}+C_{\mu} C_{e}\right)
\end{aligned}
$$

and

$$
-\frac{1}{z}=\frac{a_{1}^{\prime}}{a_{0}}=\frac{H^{\mu} \tau_{\mu}^{0}}{H^{0}}=-\frac{C_{\mu}}{g_{m}}
$$

thereby determining the transfer function exactly with much less effort and more insight that nodal analysis.

5) Source Follower With Capacitive Load: Now we consider the gain of the source-follower stage with a source resistance $R_{1}$ driving a capacitive load, as shown in Fig. $14 .{ }^{22}$ Considering $C_{\pi}$ and $C_{L}$, the ZVT's are

$$
\tau_{\pi}^{0}=r_{m} C_{\pi} \quad \tau_{L}^{0}=r_{m} C_{L}
$$

and $\tau_{\pi}^{L}$ is given by

$$
\tau_{\pi}^{L}=R_{1} C_{\pi} .
$$

${ }^{22}$ This could be the case if the stage is biased with a current source.

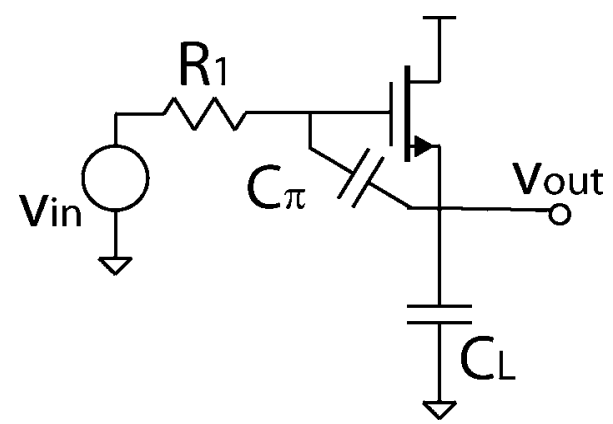

Fig. 14. Source follower stage driving a capacitive load, $C_{L}$.

In a numerical example, ${ }^{23}$ we have, $b_{1}=\tau_{\pi}^{0}+\tau_{L}^{0}=5$ ps and $b_{2}=\tau_{L}^{0} \tau_{\pi}^{L}=250(\mathrm{ps})^{2}$. Using (51) and (53), we obtain, $Q=3.16$ (about $10 \mathrm{~dB}$ of peaking) and $\omega_{n}=2 \pi \cdot 10 \mathrm{GHz}$. The fact that $Q$ is greater than 0.5 clearly indicates that we have a pair of complex-conjugate poles.

Using (40) we obtain ${ }^{24}$ an estimate for $\omega_{h}$ of $15.9 \mathrm{GHz}$, while the conventional ZVT's predict $\omega_{h}=2 \pi \cdot 32 \mathrm{GHz}$, which is twice as large. A SPICE simulations of this circuit shows $10.2 \mathrm{~dB}$ of peaking at $9.8 \mathrm{GHz}$, with an $\omega_{h}=2 \pi \cdot 15.5 \mathrm{GHz}$ all very close to the predictions obtained from our calculations. Note that in practice, this peaking is usually attenuated by $C_{\mu}$ and is not as pronounced as shown in this example. Nonetheless, the poles usually remain complex as the $Q$ is often greater than 0.5 .

6) Reactive Bandpass Filter: In this example we apply the approach to determine the exact transfer function of the reactive bandpass network of Fig. 15. The time constants are

$$
\begin{array}{rlll}
\tau_{1}^{0} & =R C_{1} & \tau_{2}^{0}=L_{2} / R & \tau_{3}^{0}=L_{3} / R \\
\tau_{2}^{1} & =2 L_{2} / R & \tau_{3}^{1}=L_{2} / R & \tau_{3}^{2}=0 \\
\tau_{3}^{12} & =L_{3} / 2 R .
\end{array}
$$

All transfer constants are zero with the exception of

$$
H^{12}=\frac{1}{2}
$$

\footnotetext{
${ }^{23}$ Assuming $C_{\pi}=C_{L}=50 \mathrm{fF}, g_{m}=20 \mathrm{mS}$ and an $R_{1}=2 \mathrm{k} \Omega$.

${ }^{24}$ The only nonzero $H^{i}$ terms are $H^{0}=1$ and $H^{\pi}=1$ leading to $a_{0}=1$, $a_{1}=r_{m} C_{\pi}$, and $a_{2}=0$
} 


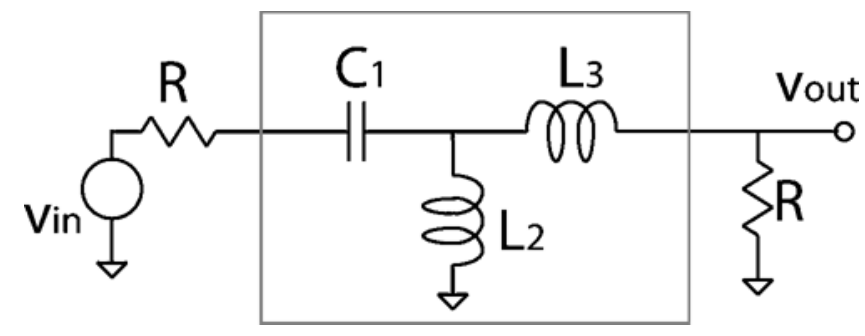

Fig. 15. Third-order reactive bandpass filter.

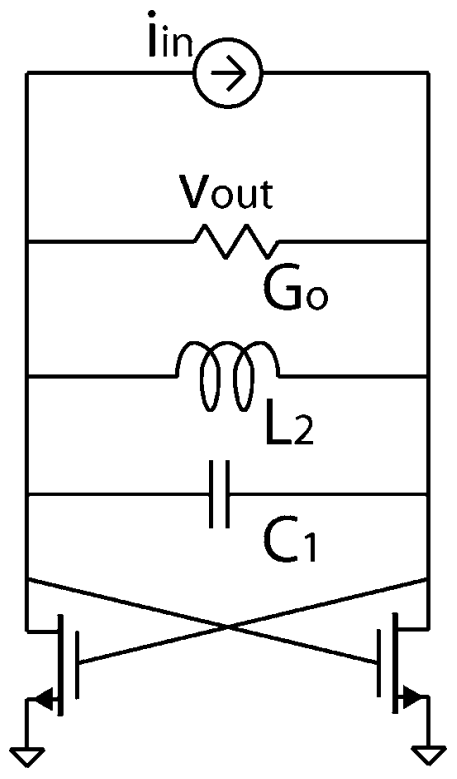

Fig. 16. Negative resistance cross-coupled oscillator.

which immediately results in the following transfer function:

$H(s)=\frac{L_{2} C_{1} s^{2}}{1+\left(R C_{1}+\frac{L_{2}+L_{3}}{R}\right) s+\left(2 L_{2} C_{1}+L_{3} C_{1}\right) s^{2}+\frac{L_{2} L_{3} C_{1}}{R} s^{3}}$

demonstrating the ease of application of the method to a passive lossless reactive network.

7) Negative Resistance: In this example, we analyze the cross-coupled NMOS pair connected across and RLC resonator, as shown in Fig. 16, where biasing details are not shown. ${ }^{25}$ Considering the input to be the differential current source, $i_{\text {in }}$, and the output to be the differential voltage, $v_{\text {out }}$, we determine the transfer function. The time constants are

$$
\begin{array}{ll}
\tau_{C}^{0}=0 & \tau_{L}^{0}=L\left(-g_{m} / 2+G_{o}\right) \\
\tau_{C}^{L}=C /\left(-g_{m} / 2+G_{o}\right) . &
\end{array}
$$

All transfer constants with the exception of $H^{L}$ are zero. Defining $G_{\text {eff }} \equiv g_{m} / 2-G_{o}$, we easily see that $H^{L}=1 / G_{\text {eff }}$. These time and transfer constants correspond to $a_{0}=0$, $a_{1}=L$, and $a_{2}=0$, as well as $b_{1}=-G_{\text {eff }} L$ and $b_{2}=L C$. Hence, we can write the transfer function as

$$
H(s) \equiv \frac{v_{\text {out }}}{i_{\text {in }}}=\frac{L s}{1-G_{e f f} L s+L C s^{2}} .
$$

\footnotetext{
${ }^{25}$ For instance, the transistors could be biased through the center tap of the inductor.
}

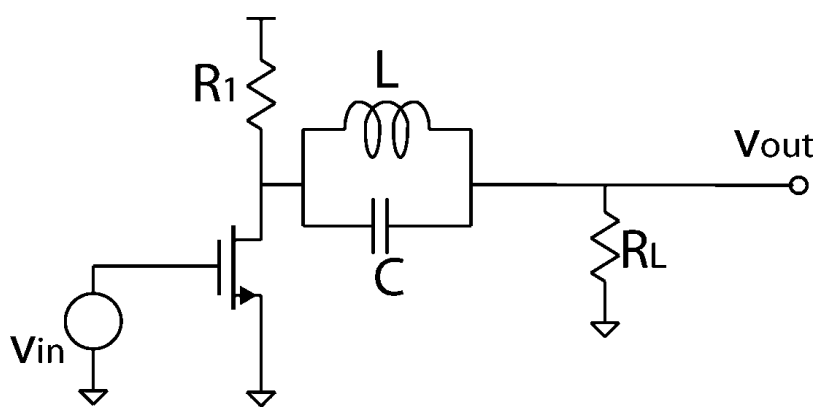

Fig. 17. Common-source amplifier with a parallel LC trap in series.

As can be easily seen, for $g_{m} / 2>G_{O}$ the denominator has a pair of RHP complex conjugate poles, corresponding to an exponentially growing response consistent with the start-up of a cross-coupled LC oscillator [18]. This example shows that the TTC approach is applicable to both stable and unstable circuits.

8) Parallel LC in Series: In the common-source amplifier of Fig. 17, we have introduced a parallel $L C$ in series. If we ignore the transistor parasitic capacitors, the ZVT's are

$$
\tau_{L}^{0}=\frac{L}{R_{1}+R_{2}}, \quad \tau_{C}^{0}=0
$$

Since $\tau_{C}^{0}=0$ and $\tau_{C}^{0} \tau_{L}^{C}=\tau_{L}^{0} \tau_{C}^{L}$ according to (26), we can avoid an indeterminant case ${ }^{26}$ by calculating $\tau_{C}^{L}=\left(R_{1}+R_{2}\right) C$ and hence

$$
\tau_{L}^{0} \tau_{C}^{L}=L C
$$

The transfer constants are

$$
\begin{aligned}
& H^{0}=H^{L}=H^{L C}=-g_{m}\left(R_{1} \| R_{2}\right) \\
& H^{L}=0
\end{aligned}
$$

which result in

$$
H(s)=H^{0} \frac{1+L C s^{2}}{1+\frac{L}{R_{1}+R_{2}} s+L C s^{2}}
$$

where according to (51), we have

$$
Q=\left(R_{1}+R_{2}\right) \sqrt{\frac{C}{L}} .
$$

As can be seen from the transfer function, there is a pair of imaginary zeros at $\pm j / \sqrt{L C}$. Since the simultaneously infinite valued inductor and capacitor result in a nonzero transfer function, $H^{L C}$, there are two zeros in the system. ${ }^{27}$

9) Input Impedance of Source Follower With Capacitive Load: Consider the source follower of Fig. 18(a), driving a capacitive load, $C_{L}$ (considering only $C_{\pi}$ and $C_{L}$ ). Calculating the input admittance, $Y(s)$, and inverting it is easier since the input impedance with both capacitors open is infinite. To calculate $Y(s)$ we must drive the input with a voltage source

\footnotetext{
${ }^{26}$ Since $\tau_{C}^{0}=0$ and $\tau_{L}^{C}=L / 0$, the product is indeterminant. If one insists on using the product $\tau_{C}^{0} \tau_{L}^{C}$, it can be determined by placing a resistor $r_{x}$ in series with $L$ and setting it to zero in the final result.

${ }^{27}$ Note that in this example, $a_{1}$ is zero while $a_{2}$ is not; thus, the zeros are a
} conjugate imaginary pair on the $j \omega$-axis. 


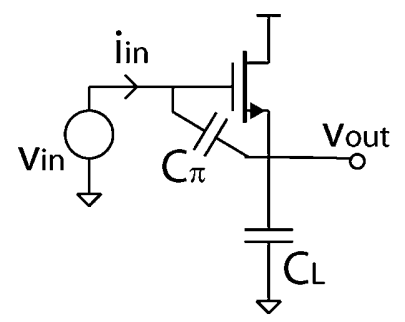

a)

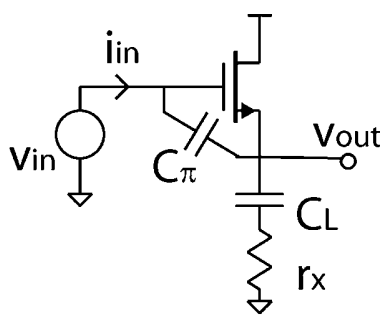

b)
Fig. 18. a) Source-follower with $C_{\pi}$ driving a load capacitor $C_{L}$; b) the same stage with an infinitesimal resistance $r_{x}$ in series with $C_{L}$.

(the stimulus) and take the input current as the output variable, as seen in Fig. 18(a).

First let us calculate $Y^{0}$ when both capacitors are open. We simply have

$$
Y^{0}=0
$$

similarly

$$
Y^{\pi}=0, \quad Y^{L}=0 .
$$

Now consider $Y^{\pi L}$. When both $C_{\pi}$ and $C_{L}$ are shorted, a short is seen looking into the input, and hence $Y^{\pi L}=\infty$. While correct this results in an indeterminant case, since for this configuration $\tau_{1}^{2}=\tau_{2}^{1}=0$ which results in a zero times infinity case for the $a_{2}$ coefficient. This can be easily resolved by introducing a resistance, $r_{x}$, (which is always there in practice anyway) in series with the $C_{L}$ (or the input) and setting it to zero later, as shown in Fig. 18(b). The previously calculated $Y^{0}, Y^{\pi}$, and $Y^{L}$ terms are still zero. The new $Y^{\pi L}$ is determined by inspection to be

$$
Y^{\pi L}=\frac{1}{r_{x}}
$$

Now to find the zero-value time constants, we see by inspection that

$$
\begin{aligned}
\tau_{\pi}^{0} & =R_{\pi}^{0} C_{\pi}=r_{m} C_{\pi} \\
\tau_{L}^{0} & =R_{L}^{0} C_{0}=\left(r_{m}+r_{x}\right) C_{L}
\end{aligned}
$$

and finally we go ahead and calculate $\tau_{L}^{\pi}$ as

$$
\tau_{L}^{\pi}=R_{L}^{\pi} C_{L}=r_{x} C_{L}
$$

Using these time-constants and low-frequency transfer functions and setting $r_{x} \rightarrow 0$, we obtain the input admittance

$$
Y(s)=\frac{r_{m} C_{1} C_{2} s^{2}}{1+r_{m}\left(C_{\pi}+C_{L}\right) s} .
$$

Note that the above expression has a single pole, because of the arrangement of Fig. 18 where $C_{\pi}$ and $C_{L}$ form a capacitive loop with the voltage source drive nulled (shorted). The above expression can be used to find the input impedance

$$
Z(s)=\frac{1}{Y(s)}=\frac{g_{m}}{C_{\pi} C_{L} s^{2}}+\frac{1}{\left(C_{\pi} \| C_{L}\right) s}
$$

which is modeled as series combination of a capacitor and what is sometimes referred to as a "super capacitor" since it has a $1 / s^{2}$ behavior [19], [20] More accurately it is a frequency dependent negative resistance (FDNR), as setting $s=j \omega$ we see that it presents a negative resistance of

$$
R=-\frac{g_{m}}{C_{\pi} C_{L} \omega^{2}}
$$

at the input. This can useful in making oscillators or filters.

\section{CONCLUSION}

The transfer function of circuits can be expressed to the desired level of accuracy in terms of time and transfer constants calculated under different combinations of shorted and opened energy-storing elements using exclusively low frequency calculations. The approach has several useful corollaries in the design of analog circuits.

\section{ACKNOWLEDGMENT}

The author would like to thank T. Arai, J. Arroyo, A. Babakhani, F. Bohn, S. Bowers, J. Chen, E. Keehr, A. Komijani, S. Kosai, S. Mehta, A. Natarajan, K. Sengupta, C. Sidiris, H. Wang, Y. J. Wang, and J. Yoo formerly or currently of Caltech for valuable feedback on the manuscript. The author would also like to thank the anonymous reviewers as well as Prof. B. Murmann of Stanford University and Prof. H. Hashemi of USC for their constructive feedback on the manuscript. Last, but not least, the author is particularly indebted to the students in Caltech's EE114 series who helped improve this material substantially over more than a decade by asking great questions and refusing to accept incomplete answers.

\section{REFERENCES}

[1] H. W. Bode, Network Analysis and Feedback Amplifier Design. New York: Van Nostrand, 1945.

[2] E. A. Guillemin, Introductory Circuit Theory. New York: Wiley, 1953.

[3] J. H. Mulligan, "The effect of pole and zero locations on the transient response of linear dynamic systems," Proc. IRE, vol. 37, pp. 516-529, May 1949.

[4] D. O. Pederson and G. H. Wilson, "Dominant zero and excess phase of a Hurwitz polynomial," IEEE Trans. Circuits Syst., vol. 11, no. 1, pp. 104-108, Mar. 1964.

[5] R. D. Thornton, C. L. Searle, D. O. Pederson, R. B. Adler, and E. J. Angelo, Multistage Transistor Circuits. New York: Wiley, 1965.

[6] B. L. Cochran and A. Grabel, "A method for the determination of the transfer function of electronic circuits," IEEE Trans. Circuit Theory, vol. CT-20, no. 1, pp. 16-20, Jan. 1973.

[7] A. M. Davis, "Analyze active-netowrk responses without complex manipulations," EDN, pp. 109-112, Feb. 1979.

[8] A. M. Davis and E. A. Moustakas, "Decomposition analysis of active networks," Int. J. Electron., vol. 46, no. 5, pp. 449-456, 1979.

[9] A. M. Davis and E. A. Moustakas, "Analyze of active RC networks by decomposition," IEEE Trans. Circuits Syst., vol. CAS-27, no. 5, May 1980.

[10] S. Rosenstark, Feedback Amplifier Principles. New York: MacMillan, 1986.

[11] R. D. Middlebrook, "Null double injection and the extra element theorem," IEEE Trans. Education, vol. 32, no. 3, pp. 167-180, Aug. 1989.

[12] R. M. Fox and S. G. Glee, "Extension of the open-circuit time-constant method to allow for transcapacitances," IEEE Trans. Circuits Syst., vol. 37, no. 9, pp. 1167-1171, Sep. 1990.

[13] P. Andreani and S. Mattisson, "Characteristic polynomial and zero polynomial with the Cochrun-Grabel method," Int. J. Circ. Theor. Appl., vol. 26, pp. 287-292, May-Jun. 1998. 
[14] P. Andreani and S. Mattisson, "Extension of the Cochrun-Grabel method to allow for mutual inductances," IEEE Trans. Circuits Syst. I, vol. 46, no. 4, pp. 481-483, Apr. 1999.

[15] R. D. Middlebrook, V. Vorperian, and J. Lindal, "The N extra element theorem," IEEE Trans. Circuits Syst. I, vol. 45, no. 9, pp. 919-935, Sep. 1998.

[16] T. H. Lee, The Design of CMOS Radio-Frequency Integrated Circuits. Cambridge, U.K.: Cambridge Univ. Press, 1998.

[17] S. Mandegaran and A. Hajimiri, "A breakdown voltage multiplier for high voltage swing drivers," IEEE J. Solid-State Circuits, vol. 42, no. 2, pp. 302-312, Feb. 2007.

[18] A. Hajimiri and T. H. Lee, "Design issues in CMOS differential LC oscillators," IEEE J. Solid-State Circuits, vol. 34, no. 5, pp. 717-724, May 1999.

[19] L. T. Bruton, "Network transfer functions using the concept of frequency-dependent negative resistance," IEEE Trans. Circuit Theory, vol. CT-16, no. 3, pp. 406-408, Aug. 1969.

[20] A. Antoniou, "Realization of gyrators using operational amplifiers and their use in RC-active network synthesis," Proc. IEE, vol. 116, pp. 1838-1850, 1969.

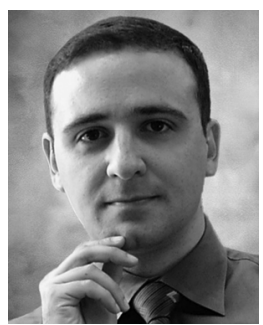

Ali Hajimiri received the B.S. degree in electronics engineering from the Sharif University of Technology and the M.S. and Ph.D. degrees in electrical engineering from Stanford University, Stanford, CA, in 1996 and 1998, respectively.

He was a Design Engineer with Philips Semiconductors, where he worked on a BiCMOS chipset for GSM and cellular units from 1993 to 1994. In 1995, he was with Sun Microsystems, where he worked on the UltraSPARC microprocessor's cache RAM design methodology. During the summer of 1997, he was with Lucent Technologies (Bell Labs), Murray Hill, NJ, where he investigated low-phase-noise integrated oscillators. In 1998, he joined the Faculty of the California Institute of Technology, Pasadena, where he is a Professor of electrical engineering and Director of the Microelectronics Laboratory. His research interests are high-speed and RF integrated circuits for applications in sensors, biomedical devices, and communication systems. He is the author of The Design of Low Noise Oscillators (Springer, 1999), and he has authored and coauthored more than 100 refereed journal and conference technical articles. He holds more than two dozen U.S. and European patents.

Dr. Hajimiri has served on the Technical Program Committee of the International Solid-State Circuits Conference (ISSCC), as an Associate Editor of the IEEE JouRnal OF SOLID-STATE CIRCUITS (JSSC), as an Associate Editor of IEEE TRANSACTIONS ON CIRCUITS AND SYSTEMS (TCAS): PART II, a member of the Technical Program Committees of the International Conference on Computer Aided Design (ICCAD), Guest Editor of the IEEE TRANSACTIONS ON MiCROWAVE THEORY AND TECHNIQUES, and on the Guest Editorial Board of Transactions of Institute of Electronics, Information and Communication Engineers of Japan (IEICE). He was selected to the top 100 innovators (TR100) list in 2004 and is a recipient of the Okawa Foundation award. He has served as a Distinguished Lecturer of the IEEE Solid-State and Microwave Societies. $\mathrm{He}$ is the recipient of Caltech's Graduate Students Council Teaching and Mentoring award as well as the Associated Students of Caltech Undergraduate Excellence in Teaching Award. He was the Gold medal winner of the National Physics Competition and the Bronze Medal winner of the 21st International Physics Olympiad, Groningen, The Netherlands. He was a corecipient of the IEEE JOURNAL OF SOLID-STATE CIRCUITS Best Paper Award of 2004, the International Solid-State Circuits Conference (ISSCC) Jack Kilby Outstanding Paper Award, a two-time co-recipient of CICC best paper award, and a three-time winner of the IBM faculty partnership award as well as National Science Foundation CAREER award. He co-founded Axiom Microdevices, Inc., in 2002, which has shipped more than 50 million fully integrated CMOS PAs and was acquired by Skywork, Inc., in 2009. 